\title{
Rupture dynamics of heterogeneous frictional interfaces
}

\author{
E. Bayart ${ }^{1,2}$, I. Svetlizky ${ }^{1}$ and J. Fineberg ${ }^{1}$
}

${ }^{1}$ The Racah Institute of Physics, The Hebrew University of Jerusalem, Jerusalem, Israel ${ }^{2}$ Univ. Lyon, ENS de Lyon, Univ. Claude Bernard, CNRS, Laboratoire de Physique, F-69342 Lyon, France

\section{Key Points:}

- Fracture mechanics describe the structure and the dynamics of rupture fronts propagating along frictional interfaces at the onset of sliding.

- A Griffith criterion is used to experimentally determine the arrest location of ruptures within highly heterogeneous interfaces.

- Rupture arrest can be due to either an abrupt change in the fracture energy, or to the value of the applied stress, when properly weighted.

Corresponding author: E. Bayart, elsa.bayart@ens-lyon.fr 


\begin{abstract}
The onset of sliding motion is conditional on the propagation of rupture fronts that detach the contacting asperities forming a frictional interface. These ruptures, when propagating over a fault surface, are the most common mechanism for an earthquake. Experimentally, the transition from static to sliding friction takes place when a rupture traverses the entire interface. But ruptures can also arrest before reaching the end of the interface. The determination of the mechanisms responsible for rupture arrest is of particular interest for understanding an earthquake's magnitude selection. Propagating ruptures have been shown to be true shear cracks, driven by singular fields at their tip and fracture mechanics have been successfully used to describe rupture arrest along homogeneous frictional interfaces. Performing high-temporal-resolution measurements of the real contact area and strain fields, we demonstrate that the same framework provides an excellent quantitative description of rupture arrest along interfaces with heterogeneous fracture properties and complex stress distributions at a macroscopic scale. This work unravels the different mechanisms responsible for rupture arrest along model laboratory-faults. This fracture-based paradigm opens a window to a wide range of possible consequences for frictional behavior along any two contacting bodies; from the centimeter scale to the scale of natural faults.
\end{abstract}

\title{
Plain language summary
}

An earthquake is a rupture front propagating along a fault, that breaks the solid contacts formed between the two fault faces. Ruptures are often arrested before spanning the entire length of the fault. Rupture lengths relate to earthquake magnitudes; the longer the rupture, the higher the magnitude. The aim of this study is to understand what causes rupture arrest, hence, what selects an earthquake's size. We mimic a fault in the lab by pressing and shearing two solid blocks until sliding motion initiates. We demonstrate that an arrest criterion derived from fracture mechanics theory is valid to determine if and where a rupture will arrest. Our conclusion is that rupture arrest occurs because of either value of the applied stress, when properly weighted, or a localized increase of the fracture energy, i.e. the energy dissipated during the rupture propagation, along the interface.

\section{Introduction}

The onset of sliding of two bodies in frictional contact is a question of interest for a broad range of phenomena and is crucial for understanding fault dynamics. Our understanding of the mechanisms at play is, however, hindered by an inherent separation of scales. Any two sliding bodies are separated by a plane of contacting micro-asperities that define a frictional interface. This ensemble of micro-asperities has a contact area that is much smaller than the (nominal) macroscopic one [Bowden and Tabor, 2001; Dieterich and Kilgore, 1996]. These micro-contacts bear the entire normal load applied to the solids, often attaining their plastic limit. The dynamics of the micro-contacts that form a frictional interface will therefore control the macroscopic response of the system.

In the stick-slip regime, i.e. an alternation of motionless loading phases and sliding phases, sliding motion is conditional on the propagation of rupture fronts that detach the contacting asperities [Rubinstein et al., 2004; Xia et al., 2004; Baumberger et al., 2003; Ben-David et al., 2010; Passelègue et al., 2013; Ohnaka, 2013]. These fronts are recognized as being the main rupture mechanism within seismic faults, and are identified with earthquakes. The framework of fracture mechanics has been widely used in geophysics [Scholz, 2002; Rice, 1980; Ben-Zion, 2008; Ida, 1972; Dunham and Archuleta, 2004; Xu et al., 2016; Xu and Ben-Zion, 2017], however, direct experimental evidence that a rupture front is a true shear crack has been provided only recently 
[Svetlizky and Fineberg, 2014]. Like shear cracks, frictional ruptures can propagate in either a sub-Rayleigh or a supershear regime [Rubinstein et al., 2004; Xia et al., 2004; Ben-David et al., 2010; Passelègue et al., 2013; Svetlizky et al., 2017a; Xu et al., 2017], leading to different types of earthquakes [Dunham and Archuleta, 2004; Peng and Gomberg, 2010]. In the former, front velocities, $C_{f}$, range from a fraction of the Rayleigh wave speed, $C_{R}$, to $C_{R}$. In the supershear regime, $C_{f}$ may exceed the shear wave speed, $C_{S}$, and approach the longitudinal speed of sound $C_{L}$. In the present work, we only consider interfacial ruptures in the sub-Rayleigh regime.

In the sub-Rayleigh regime for an interface separating materials of the same composition and approximate geometry [Aldam et al., 2016], interfacial ruptures generally accelerate towards $C_{R}$ [Freund, 1990; Svetlizky et al., 2017b] where the rate of acceleration is determined by the magnitude of the pre-existing stresses that load the system at the time of rupture nucleation. Indeed, the entire broad spectrum of rupture speed exists within relatively simple block on block frictional system [Ben-David et al., 2010; Kaproth and Marone, 2013; Bar-Sinai et al., 2013; Svetlizky et al., 2017b; $X u$ et al., 2017]. The overall sliding motion of a finite-size system only initiates when a rupture front has traversed the entire interface [Rubinstein et al., 2004]. When the applied loading is not spatially homogeneous, rupture fronts can propagate prior to the onset of motion, arresting before spanning the entire interface [Rubinstein et al., 2007; Maegawa et al., 2010; Katano et al., 2014; Braun et al., 2009; Scheibert and Dysthe, 2010; Tromborg et al., 2011; Radiguet et al., 2013; Kammer et al., 2015; Taloni et al., 2015; Bar-Sinai et al., 2015; Bayart et al., 2016a]. Arrested ruptures are of particular interest for two reasons. First, for a finite-size system, the macroscopic resistance to shear is reached solely when the overall sliding of the contacting surfaces occurs. At the macroscopic scale, this threshold is embodied in a static friction coefficient that corresponds to the stress level preceding the instance of rupture nucleation. The prediction of if and where rupture arrest will take place therefore provides a tool to predict the macroscopic failure of a system. Second, these ruptures are true analogs of earthquakes in the laboratory; an earthquake is a finite-size rupture in a nearly infinite-size system - a fault. Understanding the mechanisms of rupture arrest therefore helps us to understand the unresolved question of the selection of an earthquake's length. Different hypotheses have emerged. A widespread scenario is the cascade model, leading to the conclusion that rupture arrest is not deterministic [Brune, 1979; Ellsworth and Beroza, 1995; Kilb and Gomberg, 1999; Ellsworth and Beroza, 1998]. Another hypothesis is that the nucleation phases of large and small earthquakes are different, therefore one can know the rupture size before propagation [Ellsworth and Beroza, 1995; Beroza and Ellsworth, 1996; Kilb and Gomberg, 1999; Ohnaka, 2000; Olson and Allen, 2005], although some work suggests the opposite conclusion [Lapusta and Rice, 2003]. Barriers of energy, due to a geometrical discontinuity or to spatially heterogenous interfacial properties, have also been considered as possible causes of rupture arrest [Steacy and McCloskey, 1998; Kame and Yamashita, 1999; Ampuero et al., 2006; Wesnousky, 2006; Elliott et al., 2009]. Dynamic rupture modelling has also been used recently to evaluate the magnitude of the largest expected earthquake [Cappa and Rutqvist, 2011; Hirono et al., 2016; Lozos, 2016; Galis et al., 2017]. In this study, we investigate the reasons for rupture arrest along a heterogeneous frictional interface, i.e. with spatially varying loading distribution and/or interfacial properties.

In a recent study, inspired by the work of Kammer et al. [Kammer et al., 2015], we experimentally demonstrated how the fracture mechanics framework can be used to predict the arrest locations of partial ruptures [Bayart et al., 2016a]. There, we focused on a specific loading configuration; partial ruptures were induced by initially imposing spatially decreasing profiles of stresses along the interface. However, a number of different configurations/situations should give rise to rupture arrest. In this paper, we present experimental comparisons of a number of important such cases with theoretical predictions for rupture arrest. The paper has the following structure. After having 
introduced the experimental setup in Sec. 2, we continue with a detailed description of recent results about the structure and propagation of frictional ruptures under dry and lubricated conditions in Sec. 3. In Sec. 4, the conditions for rupture arrest are investigated, where the general framework of fracture mechanics is utilized to define these conditions. We provide a detailed description of the method used [Bayart et al., 2016a] to determine the arrest location of a frictional rupture. We then validate our method by describing the arrest of ruptures along frictional interfaces having heterogeneous fracture properties and highly non-homogeneous distributions of stresses at a macroscopic scale, i.e. the typical heterogeneity size is much larger than the asperity size and the cohesive zone size (the zone around the rupture tip where the disipative processes take place). This separation of scales justifies the applicability of the brittle fracture theory. Highly non-uniform stresses along an interface can be induced by either non-uniform loading, previous rupture history and/or spatial variations of interface toughness (here introduced by the use of partial lubrication of the interface). For these varied case studies, we will demonstrate that fracture mechanics provide an excellent description of how/why/where rupture arrest takes place in all of these highly varying scenarios. We then provide a general discussion in Sec. 5 .

\section{Experimental set-ups}

The experiments that will be described were performed using two different geometries of sliding bodies. In both types of experiment, two blocks of poly(methylmethacrylate) (PMMA) are first pressed together then sheared (Fig. 1a-b). Stick-slip behaviour ensues (Fig. 1c) and each slip event is preceded by the propagation of a rupture front (fig. 1d). This experimental setup enables us to measure the macroscopic normal and shear forces, $F_{N}$ and $F_{S}$, the real area of contact, $A(x, t)$ and the strain field along the interface. PMMA is a viscoelastic material with a rate-dependent Young's modulus, $3.2<E<5.65 \mathrm{GPa}$, also expressed in terms of shear modulus $1.2<\mu<2.1 \mathrm{GPa}$, and a Poisson ratio $\nu_{p}=0.33$. PMMA's shear and longitudinal wave speeds are $C_{S} \approx 1345 \mathrm{~m} . \mathrm{s}^{-1}$, and $C_{L} \approx 2333 \mathrm{~m} . \mathrm{s}^{-1}$ and the Rayleigh wave speed is $C_{R}=1255 \mathrm{~m} . \mathrm{s}^{-1}$ for plane strain conditions, $C_{R}=1237 \mathrm{~m} . \mathrm{s}^{-1}$ for plane stress conditions.

Asymmetric geometry. As schematically described in Fig. 1a, the top block is thin with $x \times y \times z$ dimensions $150 \mathrm{~mm} \times 100 \mathrm{~mm} \times 5.5 \mathrm{~mm}$ whereas the bottom block is thick having dimensions of $300 \mathrm{~mm} \times 30 \mathrm{~mm} \times 30 \mathrm{~mm}$ (Fig. 1a). We define $x$ as the direction of the sliding of the top block, $y$ the direction in which normal forces, $F_{N}$, are applied, and $z$ corresponds to the plate thickness dimension. The frictional interface is within the $x z$ plane. The top block is clamped at its upper edge while the bottom block is mounted on a low-friction linear translational stage. Shear forces, $F_{S}$, are uniformly applied, via the translation of this rigid stage, until stickslip motion initiates. In this configuration, the boundary conditions at the interface closely correspond to plane strain; $\varepsilon_{z z}=0$. The contacting face of the top block was diamond-machined to be optically flat. The contact surface of the bottom block was first machined to be flat and then roughened by means of a $9 \mu \mathrm{m}$ rms diamond lapping film. The experiments described were performed after numerous stick-slip events through which the initial roughness was reduced to a stable value of $0.5 \mu \mathrm{m}$ rms. Block roughness was characterized by optical profilometry [STIL CCS-Prima]. In a recent study [Aldam et al., 2016] it was shown that, at extreme propagation velocities $\left(C_{f} \sim C_{R}\right)$ this configuration becomes an effective bimaterial interface (a frictional interface separating materials with different elastic properties). In the experiments described here, we will focus on situations near or at arrest. Hence, bimaterial effects related to this asymmetric geometrical configuration can be ignored. 
a

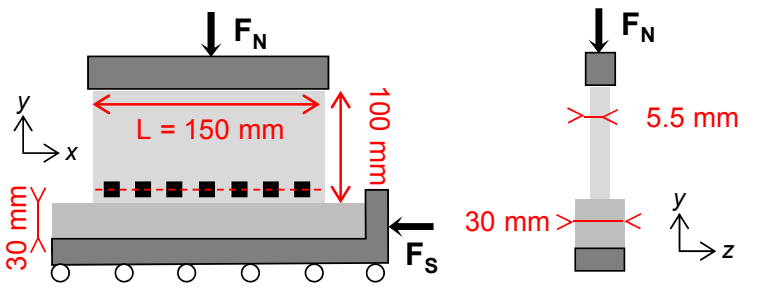

b
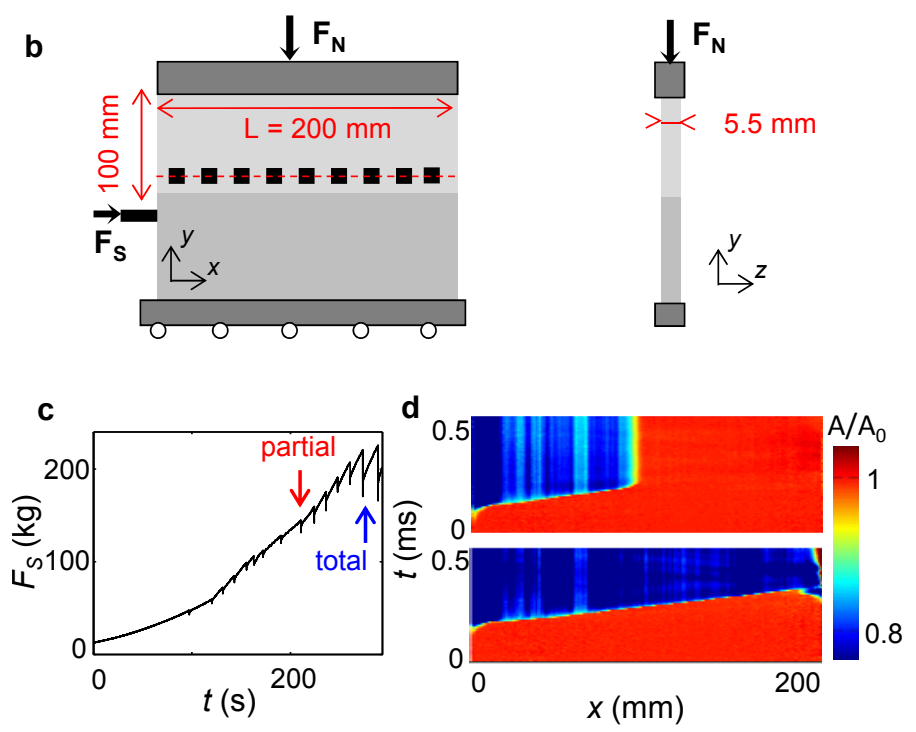

Figure 1. Experimental setup, force and contact area measurements. (a) Asymmetric and (b) symmetric geometries of two sliding blocks of PMMA. Dimensions $(x \times y \times z)$ of top and bottom blocks are respectively (a) $150 \mathrm{~mm} \times 100 \mathrm{~mm} \times 5.5 \mathrm{~mm}$ and $300 \mathrm{~mm} \times 30 \mathrm{~mm} \times 30 \mathrm{~mm}$, (b) $200 \mathrm{~mm} \times 100 \mathrm{~mm} \times 5.5 \mathrm{~mm}$ and $240 \mathrm{~mm} \times 100 \mathrm{~mm} \times 5.5 \mathrm{~mm}$. An array of (a) 14 and (b) 19 strain gages measured the strain tensor components at $y \approx 3.5 \mathrm{~mm}$ above the interface. Top blocks are clamped at their upper edges while bottom blocks are mounted on a low-friction translational stage. $F_{S}$ is applied homogeneously in (a) and at a point at the $x=0$ edge of the bottom block in (b). (c) Shear force $F_{S}$ as a function of time, $t$, of a typical stick-slip experiment using the asymmetric setup. Total denotes the first slip event (macroscopic displacement of the two blocks) while partial denotes a precursory event (arrested rupture accompanied by local slip). (d) Spatiotemporal evolution of the real contact area $A(x, t)$ for a partial (top) and total (bottom) interfacial rupture. Each line is a snapshot in time of $A(x, t)$, normalized by $A_{0}=A(x, 0)$ immediately prior to the event so as to eliminate any spatial variation of the light intensity. The rupture tips $x_{t i p}(t)$ are the locations where $A(x, t)$ drops sharply for each time $t$. 
Symmetric geometry. The symmetric geometry that we used is schematically described in Fig. 1b. This set-up consists of a frictional interface formed by two thin blocks having the same thickness. The dimensions of the top and bottom blocks are respectively $200 \mathrm{~mm} \times 100 \mathrm{~mm} \times 5.5 \mathrm{~mm}$ and $240 \mathrm{~mm} \times 100 \mathrm{~mm} \times 5.5 \mathrm{~mm}$. The top and bottom blocks were clamped respectively at their top and bottom edges. The bottom block was mounted on the low-friction translational stage, whereas the upper edge of the top block was clamped to a fixed mount. $F_{S}$ was locally applied to an edge of the bottom block via a push-rod of cross-sectional dimensions $3.5 \mathrm{~mm} \times 5.5 \mathrm{~mm}$ ( $y \times z$ directions), positioned at $-3.5 \mathrm{~mm}<y<0$ and $x=0 \mathrm{~mm} ; x=0$ is defined as the left corner of the blocks on Fig. $1 \mathrm{~b}$ and $y=0$ the contacting interface. Boundary conditions in this configuration are plain stress; $\sigma_{z z}=0$. The two contacting block faces were diamond-machined to be optically flat with $<0.1 \mu \mathrm{m}$ rms roughness. Due to the geometrical symmetry of this configuration, no effective bimaterial effects exist.

Loading. Normal loads, $F_{N}$, were quasi-statically applied at the beginning of each experiment. Shear forces, $F_{S}$, were then applied from zero by imposing a constant displacement of the translational stage at a rate of $8 \mu \mathrm{m} \cdot \mathrm{s}^{-1}$. Because of the linear geometry of the setup, rigid translation of the bottom block by the stage during the shear loading drags the bottom edge of the top block and thereby induces a torque along the interface [Ben-David and Fineberg, 2011]. Without imposing compensation for this, the torque produces a significant gradient of normal stress along the interface. We compensated for this effect by imposing a small angle $\left(\approx 0.1^{\circ}\right)$ to the top block's face relative to the bottom block prior to applying loads. The angle was chosen to provide a flat normal stress distribution over the central part of the interface at values of $F_{S}$ at which slip events took place.

Contact area measurements. The real contact area, which constitutes less than a percent of the nominal contact area under our experimental conditions, was measured using an optical technique based on total internal reflection. A sheet of light illuminated the entire interface at an incident angle whose value was well beyond the angle for total internal reflection from the PMMA to air interface. As a result, the light was entirely reflected at the interface in the absence of contacts and transmitted across the interface only at points of contact. Hence, the transmitted intensity was approximately proportional to the real contact area at each spatial point along the interface. This intensity was recorded by a camera (Phantom V710) at $580000 \mathrm{fps}$, with a spatial resolution varying between 1000 to 1280 pixels along the interface and 8 pixels along the width ( $z$ direction) of the interface. Hence, our measurements are coarse-grained, in the sense that the spatial resolution does not enable us to resolve a single contact. Each pixel in the $x$ direction maps 117 to $150 \mu \mathrm{m}$, and about 750 $\mu \mathrm{m}$ in the $z$ direction, while the roughness of the block was $0.1-0.5 \mu \mathrm{m}$, depending on the experimental setup. In the results presented, we integrated the contact area data across the $z$ direction, hence the interface is considered to be unidimensional (Fig. 1d). Prior to any frictional slip, the contact area data is stored continuously in a circular buffer of duration $13 \mathrm{~ms}$. Any Slight frictional slip along the interface excites an acoustic signal, both during the nucleation and the propagation phases. Receipt of this signal by an acoustic sensor that detected the P-waves generated by each slip event initiated the storage of the real contact area measurements in the $13 \mathrm{~ms}$ interval surrounding each slip event.

The locations where the measured light intensity drops denote the instantaneous location of the rupture front that breaks the contacts along the interface while propagating (Fig. 1d). The transmitted light is not, however, necessarily a quantitative measure of the real contact area. When considering very rough contacting surfaces, the transmitted light intensity is truly proportional to the real contact area [Rubinstein et al., 2006] while for smoother surfaces, as those used in the presented experiments, part of the intensity is transmitted by evanescent coupling of the light. In this study 

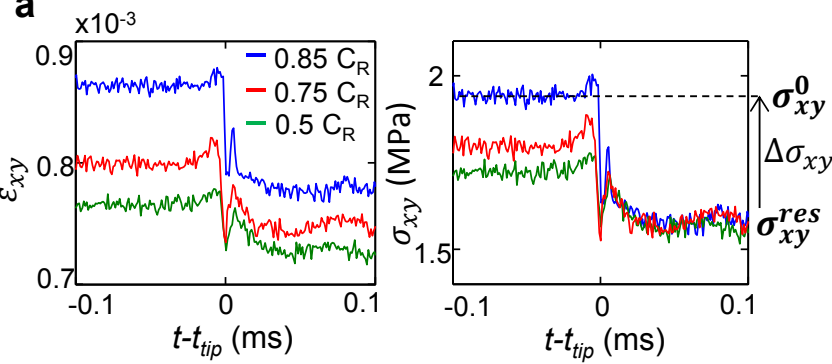

b

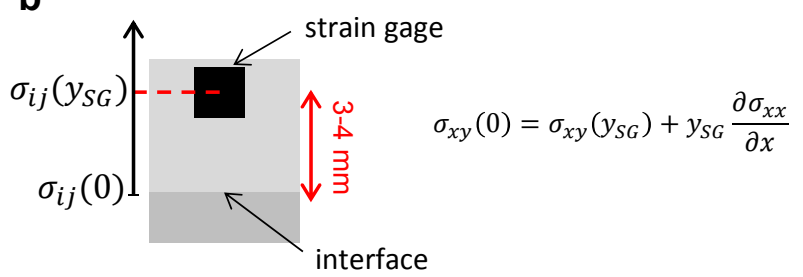

Figure 2. Conversion from strain to stress. (a) Strain measurements (left) are converted to stress (right) using the appropriate values of the Young's modulus of PMMA, a viscoelastic material (Eq. 1). Data are taken from 3 different events of a stick-slip sequence at $x=158 \mathrm{~mm}$. Differences in initial loading lead to different front velocities $C_{f}$. When viscoelastic effects are properly accounted for, residual stresses $\sigma_{x y}^{r e s}$ are nearly constant from one rupture to another at a given location. We define $\Delta \sigma_{x y}=\sigma_{x y}^{0}-\sigma_{x y}^{r e s}$, as the dynamic drop of the initial stress, $\sigma_{x y}^{0}$, induced by the rupture propagation. (b) Schematic view of a strain gage at a height $y_{S G}$ above the interface $(y=0)$. By using a first order Taylor expansion, stresses measurements performed at $y=y_{S G}$ are extrapolated to $y=0$ (Eq. 2)

only rupture tip locations provided by $A(x, t)$ will be used - as these are precise, even if $A(x, t)$ is a nonlinear function of the measured intensity.

Strain measurements. Two load cells measured the normal and shear forces at the loading points. A total of 14 (asymmetric setup) to 19 (symmetric setup) rosette strain gage arrays were mounted along the interface (Vishay 015RJ). These strain gages were mounted on one face of the top block and effectively spanned most of the interface. On the $150 \mathrm{~mm}$ block (asymmetric setup), the 14 gages were located between $x=4 \mathrm{~mm}$ and $x=144 \mathrm{~mm}$. On the $200 \mathrm{~mm}$ block (symmetric setup), the 19 strain gages were distributed between $x=5 \mathrm{~mm}$ and $x=158 \mathrm{~mm}$. Each strain gage array was composed of 3 separate strain gages aligned in a rosette configuration. Each rosette thereby provided measurements of the 3 components of the 2D-strain tensor, $\varepsilon_{i j}$, at locations situated 3 to $4 \mathrm{~mm}$ above the interface. Strain measurements were performed continuously at rates of $10^{6}$ samples/s. The strain gages needed to be calibrated using a known loading configuration to eliminate cross-talk between different measured components.

Conversion from strain to stress. The corresponding stresses, $\sigma_{i j}$, were calculated from $\varepsilon_{i j}$ after accounting for the viscoelasticity of PMMA [Bayart et al., 2016a; Svetlizky et al., 2017a]. By static loading of the blocks, we measured $E_{s}=3.2 \mathrm{GPa}$, the static Young's modulus, and using ultrasonic measurements we measured $E_{d}=5.65$ GPa, the dynamic Young's modulus, in agreement with the literature [Read and Duncan, 1981]. The stress drop immediately after the passage of a rupture tip is a rapid process that takes place within a few $\mu$ sec, a rate sufficiently high to justify the use of the dynamic Young's modulus $E_{d}$. On the other hand, the loading phase is a static 
process, and $E_{s}$ is the relevant elastic modulus at these scales. For this reason, the shear stress is related to measured strains as:

$$
\sigma_{x y}(t)=\frac{1}{1+\nu_{p}}\left[E_{d} \Delta \varepsilon_{x y}(t)+E_{s} \varepsilon_{x y}^{0}\right]
$$

where $\varepsilon_{x y}^{0}$ is the initial shear strain and $\Delta \varepsilon(t)=\varepsilon_{x y}(t)-\varepsilon_{x y}^{0}$. The same procedure was applied to calculate the two other stress components. In Fig. 2a we compare the temporal evolution of the shear strain measured at the same location for three different ruptures, within a single sequence of stick-slip events. In the figure we calculated the corresponding stress values using Eq. 1. While the initial and residual strains are different for different events, the residual stresses, $\sigma_{i j}^{r e s}$, calculated in this way were unchanged from one rupture to another. Even though we have measured a slight rate dependence of the residual stress [Svetlizky et al., 2017a], this example demonstrates the approximate constancy of $\sigma_{x y}^{r e s}(x)$ at each location along the interface, as long as the experimental conditions are unchanged. This will be discussed in Sec. 4.2.

Strain measurements, converted to stress, are performed $3.5 \mathrm{~mm}$ above the interface. When interfacial values are required, i.e. at $y=0$, extrapolation via a first order Taylor expansion of the shear stress from $y=3.5 \mathrm{~mm}$ to $y=0$ can be performed (Fig. 2b):

$$
\sigma_{x y}(0)=\sigma_{x y}\left(y_{S G}\right)+y_{S G} \frac{\partial \sigma_{x x}}{\partial x}
$$

where $y_{S G}$ is the strain gage height and we have used the equilibrium identity $\frac{\partial \sigma_{i k}}{\partial x_{k}}=$ $0 \Rightarrow \frac{\partial \sigma_{x y}}{\partial y}=-\frac{\partial \sigma_{x x}}{\partial x}$. The shear stress differences are generally minimal except in the vicinity of strong compressive stress gradients, as those produced by push-rod shear loading (Fig. 1b)

This experimental setup enabled us to quantitatively study the interfacial rupture fronts and their structure while the fronts propagated along the frictional interface at the onset of motion. In the next section, we will review recent characterizations of these fronts, both under dry and lubricated conditions.

\section{Dynamics of interfacial ruptures}

\subsection{Interfacial rupture}

When sufficiently sheared, stick-slip motion generically ensues; an alternation of slip events and motionless phases (Fig. 1c). A slip event is a short-duration macroscopic relative displacement of the two blocks. In each event, a portion of the stored elastic energy is released, inducing a drop of the applied shear force.

Each slip event is initiated by the propagation of an interfacial rupture front. The rupture fronts are easily observed in spatio-temporal plots of the contact area, recorded at times surrounding the front propagation (Fig. 1d). The contact area is reduced at rupture tips during propagation. The rupture front velocity $C_{f}$ is measured via the instantaneous space-time locations of the rupture tip, $x_{t i p} . C_{f}$ can also be determined by measuring the propagation time of the strain peak (Fig. 2a) between subsequent strain gages, although the spatial resolution is less than that of the first method. The two methods agreed on the determination of $C_{f}$. Macroscopic slip of the two blocks only ensues when a rupture front traverses the entire interface (Fig. 1d-bottom).

Under certain conditions, small drops of $F_{S}$ occur during shear loading (Fig. 1c) prior to the onset of stick-slip motion. These are induced by the propagation of interfacial ruptures that arrest before they cross the entire length of the interface (Fig. 1d-top). While these events induce local slip at points traversed by the partial rupture, macroscopic sliding of the surfaces will not occur, as the surfaces remain pinned at points beyond the rupture arrest locations. This type of event has been 
referred to in the literature as a precursor to sliding motion [Rubinstein et al., 2007; Maegawa et al., 2010; Katano et al., 2014; Braun et al., 2009; Scheibert and Dysthe, 2010; Tromborg et al., 2011; Radiguet et al., 2013; Kammer et al., 2015; Taloni et al., 2015; Bar-Sinai et al., 2015]. The mechanisms of rupture arrest will be discussed in detail in Sec. 4.

\subsection{Shear cracks}

Interfacial rupture fronts have been shown to be true singular shear cracks [Svetlizky and Fineberg, 2014]. Shear cracks are driven by singular stress fields in which the singular term is described by Linear Elastic Fracture Mechanics (LEFM) [Freund, 1990]:

$$
\begin{array}{r}
\sigma_{x x, y y}-\sigma_{x x, y y}^{0}=\frac{K_{I I}\left(C_{f}\right)}{\sqrt{2 \pi r}} \Sigma_{x x, y y}^{I I}\left(\theta, C_{f}\right) \\
\sigma_{x y}-\sigma_{x y}^{r e s}=\frac{K_{I I}\left(C_{f}\right)}{\sqrt{2 \pi r}} \Sigma_{x y}^{I I}\left(\theta, C_{f}\right)
\end{array}
$$

$r$ and $\theta$ are polar coordinates with respect to the crack tip, $\Sigma_{i j}^{I I}\left(\theta, C_{f}\right)$ are known universal angular functions for Mode $I I$ fracture and $K_{I I}$ is the stress intensity factor [Freund, 1990]. In our configuration, the system is initially loaded with shear stress $\sigma_{x y}^{0}$ and tensile stresses $\sigma_{y y}^{0}$ and $\sigma_{x x}^{0}$, where compression is denoted by positive values. After the rupture, the initial shear stress is reduced to a residual shear stress level behind the rupture tip, $\sigma_{x y}^{r e s}$. Because the LEFM equations are linear, constant values can be added to any of the fields in Eq. 3 with no loss of generality. Frictional ruptures are thereby mapped to mode II cracks defined by stress-free conditions along the crack faces by subtracting initial values, $\sigma_{y y}^{0}$ and $\sigma_{x x}^{0}$, and residual values $\sigma_{x y}^{r e s}$ from the measured values $\sigma_{i j}$ (Eq. 3) [Palmer and Rice, 1973].

The corresponding singular expression for strain variations $\Delta \varepsilon_{i j}$ can be directly compared to our strain measurements. Although performed in time, we can convert the temporal measurements to spatial coordinates using: $\varepsilon_{i j}(x, t)=\varepsilon_{i j}\left(x-\int C_{f} d t\right)$ [Svetlizky and Fineberg, 2014], where $C_{f}$ are measured at $x_{t i p}$ by means of the contact area measurements (Fig. 1d). This procedure yields measurements of $\varepsilon_{i j}\left(x-x_{t i p}\right)$ (Fig. 3).

As $C_{f}$ are known, the only fitting parameter for the 3 components of the strain tensor in Eq. 3 is $K_{I I}\left(C_{f}\right)$, the stress intensity factor. As shown in Fig. 3a, there is an excellent agreement between the singular theory and the experiments for slow fronts. The LEFM solution is derived for a steady propagating rupture. For non-steady propagation, non-singular contributions may depend on the rupture acceleration profile. Such an example is shown Fig. 3b, where, for high rupture velocities, a quantitative description of the shear component often necessitates the incorporation of nonsingular terms, accounting for the propagation of a shear wave as reported in [Svetlizky et al., 2016]. However, it has been shown that a region of K-dominance around the crack tip can always be defined [Freund and Rosakis, 1992]. The good match between measured data and steady solution (Fig. 3) shows a posteriori that such non-singular contributions can be neglected. Another condition for comparing our measurements and LEFM singular solution, is that distance between the rupture tip and the measurement point is larger than the cohesive zone size [Palmer and Rice, 1973; Freund, 1990]. This is indeed the case for our measurements as the cohesive zone size extent has been measured to be $3 \mathrm{~mm}$ [Svetlizky and Fineberg, 2014], while the measurements are taken $3.5 \mathrm{~mm}$ above the interface. The applicability of LEFM to describe the form of the strain field is not unique to PMMA, but rather general. The signature of square-root singular form can be also seen in Homalite (Fig. 4 in [Rubino et al., 2017]), Granite 


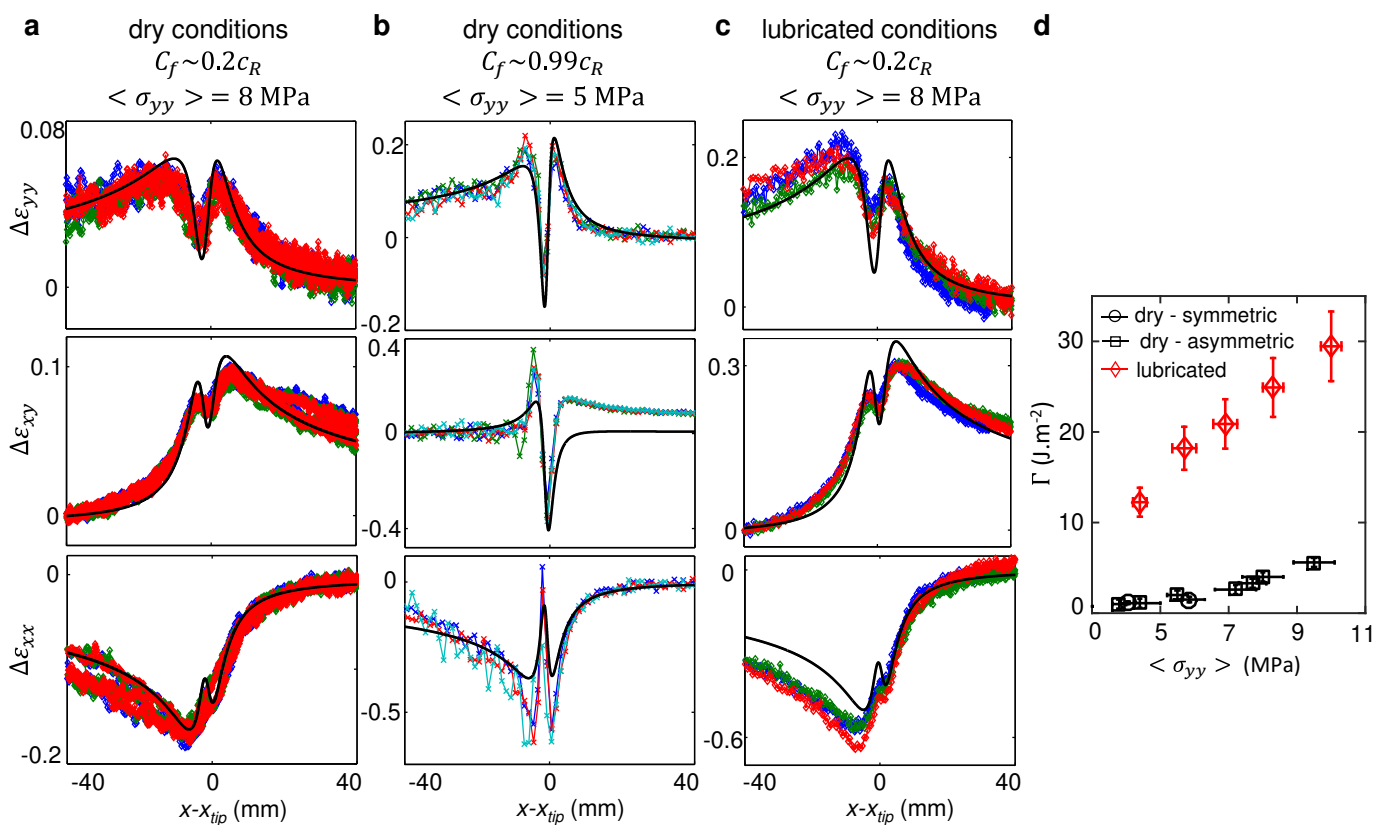

Figure 3. Dynamic strain fields and LEFM. (a-c) Variations of the strain field $\Delta \varepsilon_{i j}\left(x-x_{t i p}\right)$ with the distance from the rupture tips $x_{t i p}$, for a rupture propagating in dry friction (a) $C_{f} \approx 0.2 C_{R}$ under a normal stress $<\sigma_{y y}>=8 \mathrm{MPa},(\mathrm{b}) C_{f} \approx 0.99 C_{R}$ under a normal stress $<\sigma_{y y}>=5 \mathrm{MPa}$ and (c) in lubricated friction at $C_{f} \approx 0.2 C_{R}$ under a normal stress $<\sigma_{y y}>=8 \mathrm{MPa}$. Black solid lines are fits to the singular term of the LEFM solution for our measurement location of $y=3.5 \mathrm{~mm}$ (Eq. 3). The only fitting parameter is the fracture energy; (a) $\Gamma=2.3 \mathrm{~J} / \mathrm{m}^{2}$, (b) $\Gamma=1 \mathrm{~J} / \mathrm{m}^{2}$ and (c) $\Gamma=23 \mathrm{~J} / \mathrm{m}^{2}$. Note that the discrepancies for $\Delta \epsilon_{x y}$ from the singular term (black line in figures) at high velocities have been shown to result from higher order terms included in the full LEFM solution [Svetlizky et al., 2016] (d) Measurements of the fracture energy, $\Gamma$, versus the applied normal load $\left\langle\sigma_{y y}>\right.$ for dry $(\circ-\square)$ and lubricated $(\diamond)$ interfaces. The lubricant is a hydrocarbon oil TKO-77. 
(Fig. 1 in [Okubo and Dieterich, 1981], Fig. 10 in [Ohnaka and Kuwahara, 1990] and Fig. 1 in [McLaskey et al., 2015]) and Indian metagabbro (Fig. 9 in [Xu et al., 2017]).

We note that discrepancies between the measured $\varepsilon_{x x}$ and the theory at extreme rupture velocities $\left(C_{f}>0.96\right)$ are observed (Fig. 3b). These are not entirely understood and may result from $3 \mathrm{D}$ effects which are ignored here, a bi-material effect induced by the asymmetric geometry of the solid blocks that appears as $C_{f} \rightarrow C_{R}$ [Aldam et al., 2016] or some details of the strain gage calibration (see Supplementary Materials in [Aldam et al., 2016]). Two-dimensional effects due to tilting or geometric distortion of the rupture fronts, which are assumed to be perfectly normal to the propagation direction, might induce an averaging of the observed strain field [Fukuyama et al., 2017]. In order to minimize this effect, we limit our analysis to fronts that are straight to within a few millimeters.

\subsection{Fracture energy}

LEFM relates $K_{I I}$ to the energy release rate $G$, the flux of elastic energy per unit area of a crack's extension [Freund, 1990]:

$$
G\left(l, C_{f}\right)=\frac{1}{4 \mu\left(1-k^{2}\right)} f_{I I}\left(C_{f}, k\right) K_{I I}^{2}\left(l, C_{f}\right)
$$

where $k=C_{S} / C_{L}$ and $f_{I I}\left(C_{f}, k\right)$ is a universal function [Freund, 1990], whose value is nearly unity for low $C_{f}$. During rupture propagation, $G$ continuously balances the fracture energy $\Gamma$, defined as the dissipated energy per unit area. By using Eq. 4 coupled with energy balance, measurement of $K_{I I}$ provides a direct measurement of $\Gamma$.

$\Gamma$, the energy dissipated per unit of nominal contact area, measures the area of contacts broken during the rupture process. We verify this statement by measuring $\Gamma$ as a function of the applied normal load, $\left\langle\sigma_{y y}\right\rangle$. As the real contact area is thought to be proportional to the normal load [Bowden and Tabor, 2001], the amount of broken contacts should grow correspondingly. Values of $\Gamma$, determined by fitting the strain measurements to obtain $K_{I I}\left(l, C_{f}\right)$ and then using Eq. 4 to convert $K_{I I}$ to $G$, are indeed found to be proportional to the applied normal load, as demonstrated in Fig. 3d.

$\Gamma$ is determined with a higher accuracy for slow rupture fronts because the amplitude of the singular term is less sensitive to any experimental uncertainty in $C_{f}$ and there is no need to account for higher order corrections to the strain fields that are often necessitated at values of $C_{f}$ that approach $C_{R}$. Slow ruptures are generally observed for low dynamic stress drops $\Delta \sigma_{x y}=\sigma_{x y}^{0}-\sigma_{x y}^{\text {res }}$ [Freund, 1990; Ben-David et al., 2010; Svetlizky et al., 2017b]. We have shown [Ben-David et al., 2010; Svetlizky et al., 2017b] that over large ranges of applied stresses the interface can be either stable or unstable (under the same loading conditions). Whether the interface ruptures or not depends on whether a rupture nucleates, a process which may occur under various values of the nominal applied stresses. In other words, the macroscopic static friction coefficient, $\mu_{S}=F_{S} / F_{N}$ when a rupture is triggered, is a non-characteristic and rather ill-defined quantity. As a result, we can not in general, directly control the stress drop if nucleation is allowed to spontaneously take place, as in most of the experiments described here. Our measurements show, however, that the value of $\Gamma$ is a property that is characteristic of the interface; $\Gamma$ depends only on the interface properties and the real area of contact, i.e. the applied normal load. $\Gamma$ dependence on $C_{f}$ could potentially exist, but we have not observed it in our experiments [Svetlizky and Fineberg, $2014]$ in either dry or lubricated (see Sec. 3.4) PMMA. Hence, we can measure $\Gamma$ from experiments in which slow fronts were triggered and use this value for any experiments performed with the same applied normal load and interface conditions (see Sec. 3.4). 


\subsection{Boundary lubricated friction}

In the boundary lubrication regime, the contacting surfaces are coated by a thin lubricant layer [Hamrock et al., 2004]. In this regime, the lubricant layer does not entirely fill the contact roughness. The layer coats the asperity surfaces but the normal load is borne by the solid asperities. As in dry friction, the onset of sliding of lubricated interfaces in the boundary lubrication regime is mediated by the propagation of rupture fronts. The variations of the strain fields created by these rupture fronts are also well-described by LEFM (Fig. 3c) [Bayart et al., 2016b]. From a macroscopic point of view, a lubricant layer reduces the interfacial resistance to shear, i.e. measured friction coefficients are smaller for a lubricated interface than for a dry one. Surprisingly, the fracture energy of a lubricated interface is higher than the value of $\Gamma$ for the same non-lubricated (dry) interface. We have found that value of $\Gamma$ does not depend on the viscosity of the lubricant used but, instead, on its chemical composition [Bayart et al., 2016b]. For example, when using hydrocarbon oils, $\Gamma$ is still proportional to applied normal loads, but its value is an order of magnitude higher than for the same dry interface (Fig. 3d). We do not entirely understand the mechanism that induces this lubricant-induced toughening but believe that it might be due to either elastic coupling between the liquid layer and the solid asperity [Villey et al., 2013] or to pressure-induced crystallization of the liquid layer [Granick, 1991] .

\section{Rupture arrest}

\subsection{Definition of a rupture arrest criterion}

As mentioned in Sec. 3.1, precursors to sliding motion can occur during the shear loading phase (Fig. 4a) when the interface is only partially ruptured. Such events have been observed when a spatially inhomogeneous distribution of initial shear stress along the interface is produced by the loading [Rubinstein et al., 2007; Maegawa et al., 2010; Bayart et al., 2016a] (Fig. 5a). Such highly non-uniform stress profiles are generic along a natural fault - resulting from the existence of hard barriers, steps, fault segmentation or simply due to the previous history of the fault - as an earthquake does not always entirely relieve the stresses built up by previous events along a given fault. This rupture history dependence is also generically observed in our experiments as well - any initially imposed stress distributions will change significantly throughout a sequence of partial ruptures. Imposed stresses are only relieved when the plates are entirely separated and all of the stored elastic energy within them is allowed to be released.

Fracture mechanics has been shown to quantitatively predict rupture arrest locations [Kammer et al., 2015; Bayart et al., 2016a], if the dynamical drop of stress $\Delta \sigma_{x y}=\sigma_{x y}^{0}-\sigma_{x y}^{r e s}$ prior to rupture nucleation and the value of $\Gamma$ obtained from the dynamic stress fields are known, as described in Sec. 3.3. An implicit equation of motion of the rupture front is given when applying energy balance, $G\left(l, C_{f}\right)=\Gamma[$ Freund, 1990; Svetlizky et al., 2017b]. In particular, at the point of arrest, ruptures become quasi-static and $G_{\text {stat }}(l)=G\left(l, C_{f}=0\right)$ and $K_{I I}^{\text {stat }}(l)=K_{I I}\left(l, C_{f}=0\right)$. Energy balance then provides the arrest (Griffith) criterion [Freund, 1990]:

$$
G_{\text {stat }}=\Gamma \rightarrow K_{I I}^{\text {stat }}=\sqrt{4 \mu\left(1-k^{2}\right) \Gamma}=K_{c}
$$

where $K_{I I}^{\text {stat }}$ can be calculated from the stresses along the interface and the threshold value, $K_{c}$, is determined by $\Gamma$. A general formulation of $K_{I I}^{\text {stat }}(l)$ for a semi-infinite crack in a infinite unbounded solid, is provided by the Eshelby integral [Freund, 1990]:

$$
K_{I I}^{s t a t}(l)=\sqrt{\frac{2}{\pi}} \int_{0}^{l} \frac{\Delta \sigma_{x y}(s)}{\sqrt{l-s}} d s
$$


where $\Delta \sigma_{x y}(l)$ is a spatially dependent loading along the prospective crack path. The tip of the semi-infinite crack is initially at $x=0$ and the crack length $l$ is defined from this origin. Finite-size effects can be taken into account by choosing other formulations for calculations of $K_{I I}^{\text {stat }}$. An example suggested by [Kammer et al., 2015] shows how the effect of a free vertical boundary in $x=0$ can be incorporated in the $K_{I I}^{\text {stat }}$ calculation using a form adapted from [Tada et al., 2000]:

$$
K_{I I}^{s t a t}(l)=\sqrt{\frac{2}{\pi l}} \int_{0}^{l} \frac{\Delta \sigma_{x y}(s) F(s / l)}{\sqrt{1-(s / l)^{2}}} d s
$$

where $F(s / l)=1+0.3\left(1-(s / l)^{5 / 4}\right)$. We will show in Sec. 4.2 that the use of Eq. 6 (infinite system) or Eq. 7 (finite system) for the calculation of the $K_{I I}^{\text {stat }}$ has only a minor effect on our results. Similarly, a free boundary at $x=L$ should be accounted for. However, the last partial ruptures considered were far enough from $x=L$ (see Fig. $4 \mathrm{~b}$ for instance) so that the assumption of an infinite system holds.

The governing equation (Eq. 5) for the arrest of a rupture is $K_{I I}^{\text {stat }}=\sqrt{4 \mu\left(1-k^{2}\right) \Gamma}=$ $K_{c}$. Therefore, rupture arrest can be induced either by a local increase of $\Gamma$ or by reductions of $K_{I I}^{\text {stat }}$ due to any spatially decreasing stress drop profile, $\Delta \sigma_{x y}(x)$. Whereas the change of $\Gamma$ is entirely a local effect (e.g. induced at given locations by tougher or weaker materials along the interface), Eqs. 6 and 7 demonstrate that the value of $K_{c}$ is an integral effect of the entire stress profile over the prospective rupture path. Hence a rupture may react instantaneously to a step change in $\Gamma$ whereas a step change in $\Delta \sigma_{x y}$ will not necessarily give rise to an abrupt effect on the rupture dynamics or eventual arrest. Sufficiently low (or negative) values of $\Delta \sigma_{x y}=\sigma_{x y}^{0}-\sigma_{x y}^{r e s}$ along the interface can induce a rupture arrest. $\Delta \sigma_{x y}$ is, in turn, affected by either a decrease of $\sigma_{x y}^{0}$ or an increase of $\sigma_{x y}^{r e s}$. We note that a negative drop of stress is an interesting case where initially applied stresses are locally lower than the residual stresses, hence the rupture propagation induces a stress increase up to the residual value.

In the following, we isolate each of these three quantites $\left(\Gamma, \sigma_{x y}^{0}\right.$ and $\left.\sigma_{x y}^{r e s}\right)$ to show that each of them can be indeed responsible for rupture arrest. While all of these quantities are directly affected by the loading configuration, $\sigma_{x y}^{r e s}$ and $\Gamma$ depend also on factors such as the chemical composition of the interface layer (e.g. a lubrication layer). We first consider a homogeneous interface with decreasing initial stresses, $\sigma_{x y}^{0}(x)$, along the interface (Sec. 4.2). This configuration has been previously reported in the literature as inducing precursors to sliding motion [Rubinstein et al., 2007; Maegawa et al., 2010; Katano et al., 2014; Braun et al., 2009; Scheibert and Dysthe, 2010; Tromborg et al., 2011; Radiguet et al., 2013; Kammer et al., 2015; Taloni et al., 2015; Bar-Sinai et al., 2015; Bayart et al., 2016a]. We then consider a heterogeneous interface, where a patch of lubricant is used to impose strong variations of the constitutive properties of the interface such as $\Gamma$ (Sec. 4.3) and $\sigma_{x y}^{r e s}$ (Sec. 4.4) and demonstrate that, as predicted by the arrest criterion, the variation of each of these can indeed induce an arrest of the propagating rupture.

\subsection{Arrest induced by a spatial variation of the initial stress, $\sigma_{x y}^{0}(x)$}

In [Bayart et al., 2016a], we determined the arrest locations of partial ruptures induced by decreasing $\Delta \sigma_{x y}=\sigma_{x y}^{0}-\sigma_{x y}^{r e s}$. Shear loading was applied to the system by pushing the bottom block on one edge of the interface (Fig. 1b), resulting in initial stresses $\sigma_{x y}^{0}(x)$ that decreased with $x$ along the interface. This situation could exist within a natural fault by the existence of a hard or "immobile" barrier that pins the local plate movement. This is indeed a suggested scenario for repeating earthquakes [Rice, 1980] - which may be analogous to the sequence of arrested ruptures presented in Fig. 4. In this section, we review the results presented in [Bayart et al., 2016a] while providing additional details on the implemented methods. 

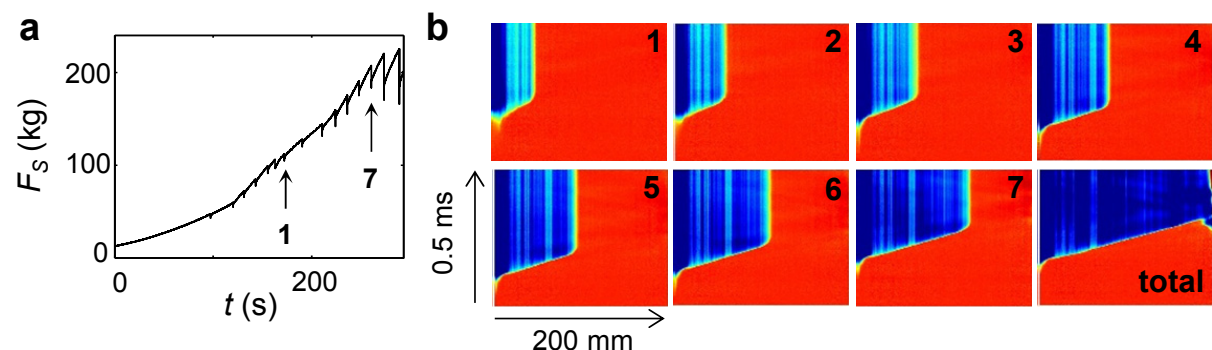

Figure 4. A sequence of arrested ruptures that took place prior to a stick slip event. (a) The applied shear force $F_{S}$ as a function of time, $t$, in a typical experiment where shear loading is applied with a push-rod at $x=0$ (Fig. 1b). (b) Contact area measurements reveal a series of partial ruptures (1-7) preceding a typical sliding event (total) along a dry interface. The sequence of 7 events bracketed by events 1 and 7 denoted in (a) are shown.

Our goal is to determine arrest locations for a series of arrested ruptures by applying the Griffith criterion. We will then compare these predictions to the actual arrest locations, as optically determined from our contact area measurements. We first consider the sequence of arrested ruptures that are generated by the loading curve presented in Fig. 4a. We consider only the last 7 arrested ruptures, as the initial ones were too short to be accurately predicted. Each event corresponds to a partial rupture of the interface (Fig. 4b); the larger the applied shear force, the longer the rupture. The rupture of the entire interface in panel 8 of Fig. $4 \mathrm{~b}$ is labeled total.

To apply Eq. 5, we need to determine both $\Gamma$ and $K_{I I}^{\text {stat }} . \Gamma$ is measured using the dynamic strain fields of slow ruptures (Fig. 3) and $K_{I I}^{\text {stat }}$ is calculated using Eqns. 6-7 once $\Delta \sigma_{x y}=\sigma_{x y}^{0}-\sigma_{x y}^{r e s}$ is known. The initial and residual stresses, $\sigma_{x y}^{0}$ and $\sigma_{x y}^{r e s}$, are obtained from strain measurements by accounting for the viscoelasticity of the PMMA, as described in Sec. 2. We note that the initial stresses, $\sigma_{x y}^{0}(x)$ can be strongly influenced by the rupture dynamics that take place within each of the prior rupture events. For example, each arrested rupture gives rise to a singular stress field surrounding the arrested location [Radiguet et al., 2013]. The profile created by each arrested event therefore significantly modifies the stress profile for each successive event. We demonstrate how the local spatial profiles of $\sigma_{x y}^{0}(x)$ are significantly influenced by a succession of precursory events in Fig. 5a. In order to use Eqns. 6-7, $\sigma_{x y}^{r e s}$ should be determined along the entire interface while it is only known where the rupture has already propagated, i.e. until the arrest location. To address this issue, we should understand what determines the value of $\sigma_{x y}^{r e s}$.

The residual stress, $\sigma_{x y}^{r e s}$ is, to first order, proportional to the applied normal stress as usually depicted by the dynamic friction coefficient (Fig. 5b). In PMMA we found [Svetlizky et al., 2017a] that $\sigma_{x y}^{r e s}$ has an additional relatively weak dependence on the material sliding velocity. This dependence can be, however, quite stronger in other materials [Rubino et al., 2017]. $\sigma_{x y}^{r e s}$ is also dependent on other parameters such as loading history, cleaning procedure or surface geometry. Here, we only consider rupture events within the same stick-slip sequence, so such effects will not influence our results. Under these conditions, we find that the residual shear stresses throughout an entire stick-slip sequence, including the partial ruptures, are effectively constant at each location along the interface (Fig. 5c).

The sole exception to the invariance of $\sigma_{x y}^{r e s}(x)$ takes place near $x=0$, where extreme normal $\left(\sigma_{y y}\right)$ and extensional $\left(\sigma_{x x}\right)$ stress variations (hence $\sigma_{x y}^{r e s}$ variations) due to shear-induced rotation of the top block are observed. These rotations are caused 

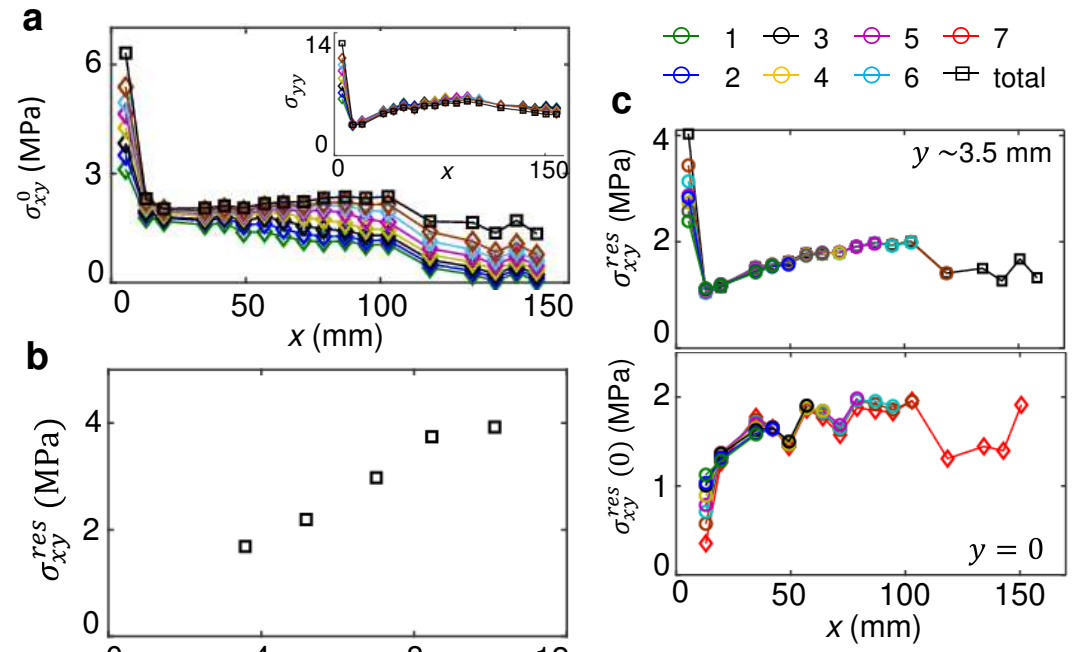

Figure 5. Initial and residual stresses induced by successive arrested ruptures that occurred prior to a single stick slip event. (a) Initial shear stress profiles $\sigma_{x y}^{0}(x)$ for the 8 events presented in Fig. 4. Loading is locally applied in $x=0$, inducing a concentration of stresses at this point. The interface is continuously loaded from one arrested rupture event to another. (inset) Normal stress profiles, $\sigma_{y y}(x)$, for each events are nearly not influenced by the arrested rupture sequence. The only significant variations occur near the $F_{S}$ loading point at $x \sim 0$. The variation of $\sigma_{y y}$ near $x=0$ is due to shear-induced rotation of the top block (Sec. 2). (b) Residual stress $\sigma_{x y}^{\text {res }}$ as a function of the local normal stress $\sigma_{y y}$, at a typical location $(x=93 \mathrm{~mm})$, from different experiments where $\sigma_{y y}$ were systematically varied. To first order, $\sigma_{x y}^{r e s}=\mu_{d} \sigma_{y y}$, $\mu_{d}$ being the dynamic friction coefficient. (c) Top - Residual shear stress profiles $\sigma_{x y}^{r e s}(x)$ along the interface for the 8 events presented in Fig. 4. Note that although the residual stress beyond the arrest location of each partial rupture is not defined, all measured values are in excellent agreement with the values of the system-wide "total" event. Bottom - Extrapolated $\sigma_{x y}^{r e s}(x)$ at $y=0$ for events 1-7 and total using Eq. 2. After this correction is applied, $\sigma_{x y}^{r e s}(x)$ is very constant from one event to another, except at the corner $x=0$ where high stress gradients exist. 

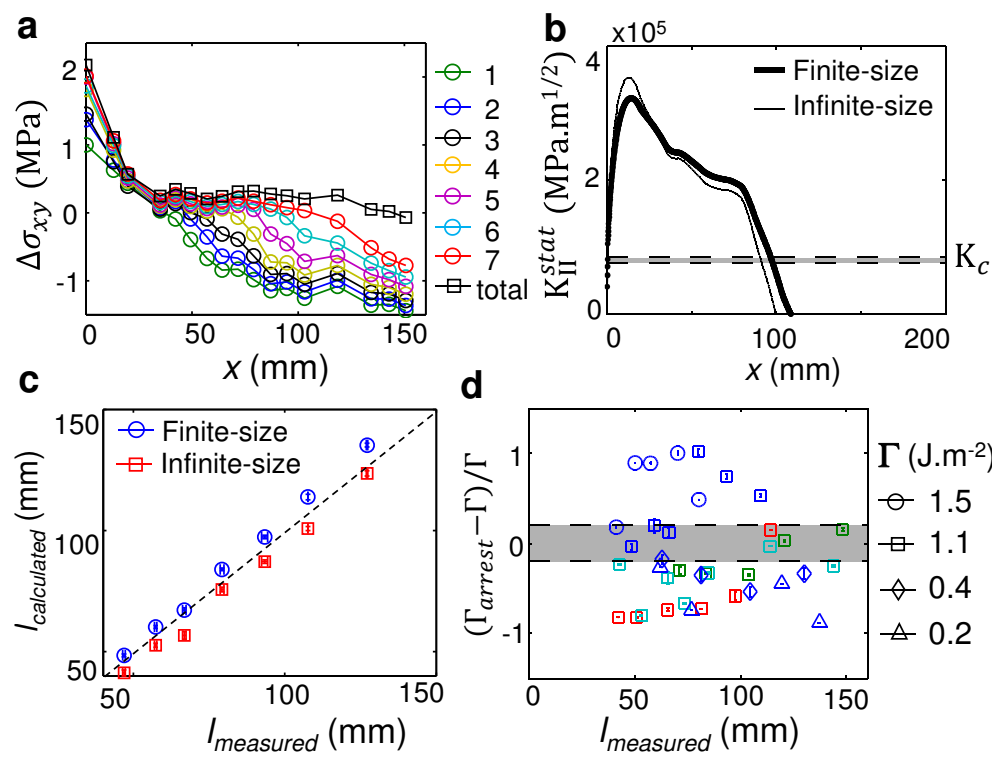

Figure 6. Determination of rupture arrest locations using fracture mechanics. (a) Shear stress drops $\Delta \sigma_{x y}=\sigma_{x y}^{0}-\sigma_{x y}^{r e s}$, along the interface for each of the ruptures presented in Fig. 4 . Stresses are plotted for $y=0$ and data are linearly extrapolated near $x=0$ (see Sec. 4). (b) The computed static stress intensity factor $K_{I I}^{\text {stat }}(x)$ for rupture 5 in Fig. 4 a. The thin line is for an infinite size system (Eq. 6) whereras the thick line is for a finite size system with a free boundary in $x=0$ (Eq. 7). $K_{c}$ (dashed line) is determined via Eq. 5 and the measured values of $\Gamma$. (c) Comparison of the measured arrested rupture lengths, $l_{\text {measured }}$, as determined from the contact area measurements to the predicted lengths, $l_{\text {calculated }}$, computed in (b) for both infinite $(\square)$ and finite (o) size systems. The dashed line has a slope 1 and is a guide to the eye. (d) Estimates of $\Gamma$ by use of the arrest locations $x=l_{\text {measured }}, \Gamma_{\text {arrest }}$. The gray area denotes the uncertainty in our $\Gamma$ determinations performed by fitting the dynamic strain field. The dynamic measurements are much more accurate although the two measurement methods for $\Gamma$ are consistent with one another. 
by the different $\sigma_{x y}^{0}$ levels required to nucleate each rupture. This effect prevents us from using a unique profile of $\sigma_{x y}^{r e s}(x)$ for any rupture events. For each event, $\sigma_{x y}^{r e s}(x)$ is measured along the rupture path. As shown in Fig. 5c, away from $x=0$, the interface residual stress is extremely well defined at each location, $x$, and constant from one rupture event to another. For this reason, beyond the arrest location, the residual stress profiles are completed with the measurements taken from the total rupture of the interface. Additionally, the shear stress measurement extrapolation from $y=3.5$ $\mathrm{mm}$ to $y=0$ is performed (Sec. 2). This correction was applied to both $\sigma_{x y}^{0}$ and $\sigma_{x y}^{r e s}$ to determine $\Delta \sigma_{x y}$ (Fig. 6a). Near the system's edge, we used a linear extrapolation of the spatially discrete measurement values of the stress drop near the edge to perform the integral calculations in Eqs. 6 or 7 (the use of alternative extrapolation functions produced a negligible influence on our results).

The static stress intensity factor $K_{I I}^{s t a t}$ is determined by integrating $\Delta \sigma_{x y}(x)$ along the prospective path of the rupture, using either the Eshelby integral (Eq. 6) or the finite-size formulation (Eq. 7). As Fig. 6b shows, the predicted arrest location is defined at the crossover between $K_{I I}^{\text {stat }}(l)$ and the Griffith criterion (Eq. 5), $K_{c}=$ $\sqrt{4 \mu\left(1-k^{2}\right) \Gamma}$. The rupture length determined by this method, $l_{\text {calculated }}$, is compared to the one observed on the contact area measurements, $l_{\text {measured }}$, for the 7 partial ruptures. Fig. 6c reveals excellent agreement between these quantities. The choice of the $K_{I I}^{\text {stat }}$ formulation has only a minor effect on the values of $l_{\text {calculated }}$.

We may also use the determination of $K_{I I}^{\text {stat }}$ at the rupture arrest as a method to measure $\Gamma$. In Fig. $6 \mathrm{~d}$ we present measurements of $\Gamma$ by this method for 37 ruptures extracted from 7 different experiments. The values of $\Gamma$ obtained by this method, $\Gamma_{\text {arrest }}$, are in agreement with the directly measured values, however, their accuracy is only within about $100 \%$. This is partially due to the fact that, in this experimental configuration, $K_{I I}^{\text {stat }}$ sharply drops near the arrest location (Fig. 6b) and therefore, any uncertainty in the arrest location, on the stress drop at the arrest location or on the choice of the $K_{I I}^{\text {stat }}$ formulation gives rise to a large uncertainty in the value of $K_{I I}^{\text {stat }}$ and therefore in the $\Gamma$ value produced. This method, however, could provide a more accurate characterization of $\Gamma$ when weaker spatial gradients are induced by the loading.

The results presented above describe partial ruptures propagating along a dry and spatally homogeneous interface. The same framework, however, can be used to describe the dynamics of partial ruptures along highly heterogeneous interfaces. In Sec. 4.3 and 4.4, we utilize selective interface lubrication to strongly modify the interface properties. Under these conditions, we demonstrate our method's validity when considering rupture propagation under highly complex stress distributions and interface conditions. In both cases, the heterogeneity size is comparable to the interface's length $(150-200 \mathrm{~mm})$, and is therefore much larger than the asperity size ( $\mu \mathrm{m}$ scale) and the cohesive zone size (mm scale). Hence, the conditions for the application of the LEFM framework are satisfied.

\subsection{Barrier of fracture energy $I$}

We have shown that a lubricant layer, in the boundary lubrication regime, does not modify the rupture mechanism but increases the fracture energy, $\Gamma$ (Fig. 3c-d). In this section, we show that a patch of lubricant can indeed prevent rupture propagation, when a rupture that is nucleated in the dry section approaches the lubricated section. Application of a lubricant on only part of the interface, the other part being dry, creates step function of increased $\Gamma$ and therefore increased $K_{c}$. 

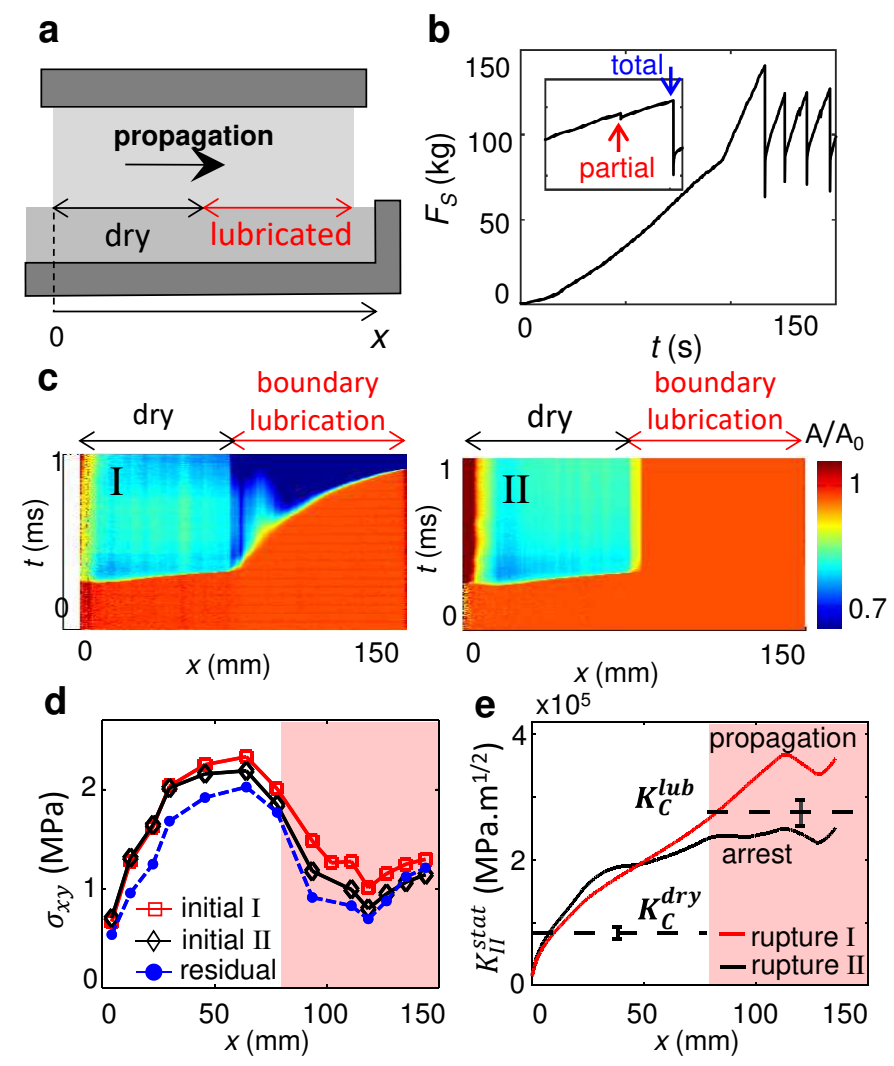

Figure 7. Rupture arrest induced by an imposed barrier of fracture energy. (a) Using the asymmetric system (Fig. 1a), half of the interface is covered with a thin layer of lubricant (TKO77). Ruptures nucleated within the dry section, at $x=0$. (b) The loading curve, $F_{S}$ as a function of time $t$. The inset is an expanded view of the third slip event in which the partial rupture shown in (e) induced a small drop of force. (c) Contact area measurements of (I) a rupture which traversed the entire interface and (II) one which arrested at the border between dry and lubricated interface. (d) Initial shear stress profile, $\sigma_{x y}^{0}(x)$, preceding ruptures I ( $\left.\square\right)$ and II $(\diamond)$ and residual shear stress, $\sigma_{x y}^{r e s}(x)$, of rupture I measured at $y \approx 3.5 \mathrm{~mm}$. Note that the extrapolation of shear stresses from $y \approx 3.5 \mathrm{~mm}$ to $y=0$ (Eq. 2) only introduces a small correction as spatial gradient of $\sigma_{x x}$ are weak in this configuration. (e) Computed static stress intensity factors, $K_{I I}^{\text {stat }}(l)$, for rupture I (red line) and II (black line). The horizontal dashed lines correspond to the Griffith criteria of dry, $K_{c}^{d r y}$, and lubricated, $K_{c}^{l u b}$, sections. At the border between dry and lubricated sections, $K_{I I}^{\text {stat }} \geq K_{c}^{\text {lub }}$ for rupture I, while $K_{I I}^{\text {stat }}<K_{c}^{\text {lub }}$ for rupture II. The white and red backgrounds of the graphs correspond to, respectively, the dry and lubricated parts of the interface. 


\subsubsection{Experimental methods}

We used the asymmetric setup in which the bottom block was homogeneously sheared (Fig. 1a). A thin layer of hydrocarbon oil was applied between $x=73 \mathrm{~mm}$ and $x=150 \mathrm{~mm}$ (Fig. 7a). The thickness of the lubrication layer used was much less than the typical asperity size $(<0.5 \mu \mathrm{m})$ and far less than that required to fill the rough interface. Under these conditions pore pressure effects are not important. Measurements of the contact area and strain at 14 different locations along the interface were performed as presented in Sec. 2. The applied normal force was $F_{N}=3500 \mathrm{~N}$, giving rise to a mean normal stress $\left\langle\sigma_{y y}\right\rangle=4 \mathrm{MPa}$.

We note that the optical coupling of lubricated surfaces is larger than that of dry surfaces. As the refractive index of the lubricant is close to that of the PMMA (see Supplemental Materials in [Bayart et al., 2016b]), this enhanced signal could be due to:

(i) increased contact area as the lubricant fills up the gaps within contacting asperities,

(ii) the presence of capillary bridges that would transmit the light without significantly increasing the interfacial strength,

(iii) increased evanescent coupling as the effective separation distance between surfaces is decreased.

For any combination of these reasons, in Fig. 7c the light intensity reduction at rupture tips within the lubricated section of the interface is larger than within the dry part. While this enhanced optical reduction does not provide a quantitative estimate of the amount of broken contacts, we can still use the $A(x, t)$ signal to easily identify the location of the rupture front throughout each experiment.

\subsubsection{Results}

Once the system is sufficiently sheared, stick-slip motion ensues (Fig. 7b). A main slip event is sometimes preceded by a small drop of $F_{S}$ (inset of Fig. $7 \mathrm{~b}$ ), induced by a partial rupture of the interface (Fig. 7c). In this series of experiments, all of the ruptures spontaneously nucleated at $x=0$, at the corner of the dry interface. When propagating rupture fronts encountered the lubricated interface, they either slowed down when traversing the lubricated section (rupture I) or entirely arrested (rupture II). We will show that, in the case of rupture II, the lubricant acts as a barrier for the rupture propagation by locally increasing $\Gamma$.

Initial stresses, $\sigma_{x y}^{0}$, were measured for the two ruptures together with the residual stresses, $\sigma_{x y}^{r e s}$ (Fig. $7 \mathrm{~d}$ ). We note that both $\sigma_{x y}^{0}$ and $\sigma_{x y}^{r e s}$ are significantly reduced within the lubricated section. As in Sec. $4, \sigma_{x y}^{r e s}(x)$ was unchanged in the dry section for partial and total rupture events. Rupture I was therefore used to measure $\sigma_{x y}^{r e s}(x)$. It is important to note that, in both cases I and II, despite the significant stress reductions within the lubricated patch, initial stresses were all higher than the residual stress, unlike the example in Sec. 4.2. We performed the $K_{I I}^{\text {stat }}$ calculation as presented in Eq. 7. Fig. 7d shows that, in case of propagation (rupture I), $K_{I I}^{\text {stat }}>K_{c}^{\text {lub }}$ when the rupture encountered the edge of the lubricated patch, at $x=73 \mathrm{~mm}$. In contrast, $K_{I I}^{\text {stat }}<K_{c}^{\text {lub }}$ for rupture II. Hence the rupture could not propagate further and arrested immediately at the patch's edge.

Here, the patch of lubricant acted as a barrier for the rupture propagation. Although this is perfectly understandable in terms of fracture mechanics (as explained above), it seems to be counter-intuitive - as the lubricant reduces the interfacial resistance to shear. Macroscopically, the friction coefficients are indeed reduced; $\mu_{s}$, the ratio of the shear force and the normal force at the onset of sliding, was reduced from 
0.56 (dry interface) to 0.44 under identical external loading conditions. This dramatic change, however, has no effect on the rupture propagation condition. As Eqs. 6 and 7 show, only the integrated stress difference, $\Delta \sigma_{x y}(x)$, controls rupture dynamics. This can be seen in the different dynamics of ruptures I and II. A slightly higher initial stress in the dry part of the interface enabled rupture I to traverse the energy barrier, while rupture II could not overcome the increased $\Gamma$ at the edge of the patch - despite that fact that $\Delta \sigma_{x y}$ was everywhere greater than zero.

We would like to stress that our focus here is on the condition of propagation at the border between the dry and lubricated sections. Concerning the propagation beyond this point, the rupture velocity in the lubricated patch is governed by the equation of motion, defined by the balance of the energy flux, given by Eq. 4, with the local energy dissipation, $\Gamma(x)$ [Svetlizky et al., 2017b]. For rupture I (Fig. 7c), the level of $\sigma_{x y}^{0}(x)$ when the rupture is nucleated, coupled to $\sigma_{x y}^{r e s}(x)$ and the local $\Gamma$, result in a slowing down of the rupture in the lubricated section consistent with the increase of $\Gamma(x)$ within the lubricated patch.

The rather surprising fact that a lubricated patch may significantly increase fracture toughness despite having a weaker resistance to shear, may be particularly relevant for fault dynamics. For example, a patch of low stress resistance along a natural fault, such as melted or water-impregnated rocks, could conceivably increase the local fracture toughness and arrest the rupture propagation, thereby resulting in shorter earthquake propagation distances than within a fault whose interface properties are uniform. These results demonstrate that interface toughness is a key parameter with which to understand rupture length selection.

\subsection{Barrier of residual stress}

In this section, we again consider a partially lubricated interface, i.e. part is lubricated and part is dry, that generates a heterogeneous spatial distribution of residual stress (Fig. 8a). The difference with the previous experiment (Sec. 4.3) is that we force nucleation to occur within the lubricated section by utilizing a thick layer of lubricant, i.e. increasing the pore pressure compared to the dry section. This results in the generation of arrested rupture events during the shear loading phase (Fig. 8b). We demonstrate that, here, the reason for rupture arrest is the highly heterogeneous spatial distribution of residual stress. Our results provide an understanding of how homogeneous loading can dynamically generate a highly inhomogeneous stress profile, which eventually leads to failure of the entire interface.

\subsubsection{Experimental methods}

Using the asymmetric setup (Fig. 8a), in which shear is homogeneously applied, we inundated part of the interface $\left(0<l_{\text {oil }}<L\right)$ with a thick layer of hydrocarbon oil TKO-77 that, in contrast to the lubricant layer used in the previous section, entirely filled the interface roughness, altogether forcing out the air gaps that exist within interfaces in both the dry and boundary lubrication cases described previously (Secs. 3). In this experiment, we use this thick layer of lubricant to selectively trigger rupture nucleation within the lubricated section. The applied normal force was $F_{N}=6000$ $\mathrm{N}$. The existence of a lubricant layer that filled the rough interface prevented us from performing contact area measurements within the lubricated part due to the optical index matching of the lubricant and the PMMA (Fig. 8c), i.e. all of the incident light was transmitted through the interface, as no total internal reflection could take place. 
a

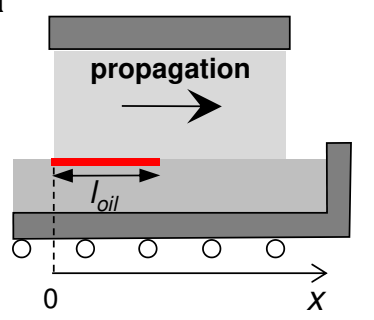

b

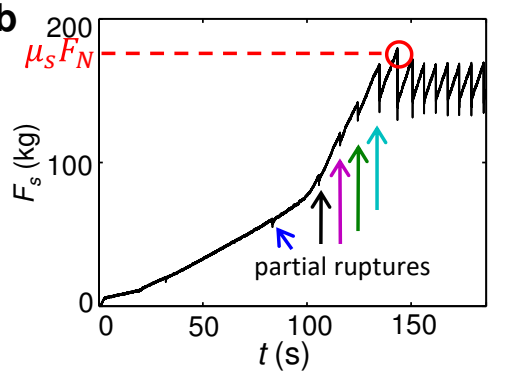

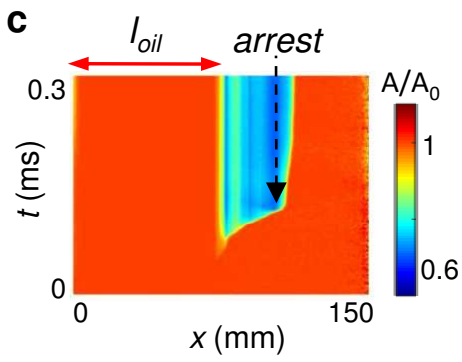

Figure 8. Rupture arrest induced by increased residual stresses. (a) Using the asymmetric setup, a thick layer of lubricant (TKO-77) of length $l_{\text {oil }}$, is applied along part of the interface. This thick layer of lubricant entirely fills the rough interface and thereby reduces $\sigma_{y y}$ via resulting pore pressure. Ruptures therefore nucleated in the oil patch, at $x=0$. (b) Shear force $F_{S}$ as a function of time $t$ for an experiment where $l_{o i l}=75 \mathrm{~mm}$. Partial ruptures within the interface occur before the onset of sliding. The static friction coefficient, $\mu_{S}$, denotes the value of $F_{S}$ when overall sliding motion starts. (c) Contact area measurements of the rupture denoted by the green colored arrow in (b) which nucleated within the lubricant layer that progressed into the dry section of the interface before arresting. Note that the rupture cannot be seen within the thick layer of lubricant because the lubricant filled the rough interface and its index of refraction is approximately equal to that of PMMA. Hence, there is no total internal reflection in non-contacting regions of the lubricated interface.
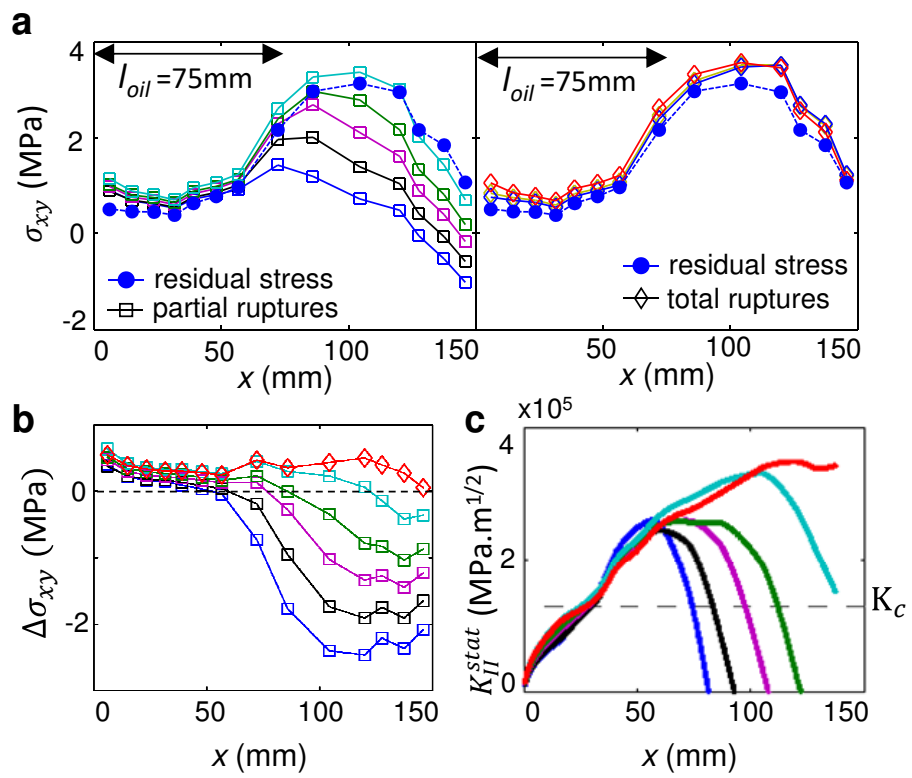

Figure 9. Rupture arrests induced by increased residual stresses. (a) The initial shear stresses profiles, $\sigma_{x y}^{0}(x)$, that are built up by successive rupture events for (left) partial ruptures and (right) total ruptures at $y \approx 3.5 \mathrm{~mm}$. The residual stress, $\sigma_{x y}^{\text {res }}(\bullet)$, used in these experiments is measured from the first total rupture of the interface. (b) The drop of stress, $\Delta \sigma_{x y}(x)=\sigma_{x y}^{0}-\sigma_{x y}^{r e s}$, along $x$ for the 5 partial ruptures $(\square)$ and the first total event $(\diamond)$. (c) The static stress intensity factor, $K_{I I}^{\text {stat }}$ as a function of $x . K_{c}$ denotes the critical stress intensity factor, $K_{c}=\sqrt{4 \mu\left(1-k^{2}\right) \Gamma}$. As in Fig. 6, rupture arrests indeed took place when $K_{I I}^{\text {stat }}=K_{c}$. 


\subsubsection{Results}

In such a heterogeneous system, numerous partial ruptures precede the macroscopic failure of the frictional interface (Fig. 8b). In this case, the partial ruptures propagate within the lubricated patch and arrest after impinging upon the dry section (Fig. 8c). As in the experiment of Sec. 4.2, successive ruptures increase in length within the dry section, until total rupture of the interface takes place.

In order to understand what caused the arrest, we measured both $\sigma_{x y}^{0}(x)$ and $\sigma_{x y}^{r e s}(x)$ (obtained from the total rupture) (Fig. 9a). As seen in Sec. 4.3, the lubricant strongly reduces both $\sigma_{x y}^{0}$ and $\sigma_{x y}^{r e s}$. In particular, the thick layer of lubricant reduces $\sigma_{x y}^{r e s}$ to almost 0 , while $\sigma_{x y}^{r e s}$ is rather high within the dry part of the interface. As a consequence, the residual stress distribution is very inhomogeneous due to the lubricated patch. The measurements of $\Delta \sigma_{x y}$ (Fig. 9b) together with Eq. 7 enabled us to calculate $K_{I I}^{\text {stat }}$ (Fig. 9c). In the oil patch, $\Delta \sigma_{x y}>0$ is reached for low values of $F_{s}$. This results in a sequence of partial ruptures that propagate across the entire lubricated sections and arrest within the dry section, where $\Delta \sigma_{x y}<0$ (Fig. 9b). The effect of the (invisible) rupture fronts that propagate within the lubricated section is seen in the strain measurements, where the signature of the singular field formed at the rupture tip location can be followed from one strain gage to another. Ruptures are "invisible" in the contact area measurements of the lubricated section since the refractive indices of PMMA and the lubricant are nearly matched (Fig. 8c) so no total internal reflection can take place within the lubricated section of the interface. The sharp increase of $\sigma_{x y}^{r e s}$ at the boundary separating the lubricated and dry interfaces at $x=75 \mathrm{~mm}$ acts as a barrier for rupture propagation as it strongly reduces $\Delta \sigma_{x y}$.

We note that the succession of partial ruptures is the process through which a frictional interface with a heterogeneous stress distribution is loaded until failure. Each successive rupture induces a concentration of stress ahead of its arrest location due to the singularity formed at its tip [Radiguet et al., 2013]. This facilitates sequentially longer rupture propagation in successive events as, between ruptures, $\sigma_{x y}^{0}$ has been locally increased. For each arrested rupture, however, the values of $\sigma_{x y}^{0}(x)$ are still below $\sigma_{x y}^{r e s}$. As a result, $\Delta \sigma_{x y}<0$ and, therefore, the condition $K_{I I}^{s t a t} \geq K_{c}$ can not be fulfilled throughout the entire dry section of the interface, and the rupture arrests. By this process, the dry section of the interface becomes increasingly loaded while stresses are intermittently released within the lubricated part (Fig. 9). This "inch-worm" scenario, which is shown here explicitly, was first suggested intuitively in [Rubinstein et al., 2009].

\section{Discussion}

We have shown how fracture mechanics governs the interfacial rupture dynamics at the onset of frictional motion. Interface ruptures are mapped to true shear cracks; they are characterized by singular fields at their tips and their motion is quantitatively described by the classic Mode II equation of motion which is governed by energy balance. The equation of motion is also successful in defining interfacial rupture arrest.

Energy balance incorporates the effects of the loading, in both $\sigma_{x y}^{0}(x)$ and $\sigma_{x y}^{r e s}(x)$, and the dissipation at the rupture tip, $\Gamma$. These quantities in bulk materials are generally simple to understand; fracture energies are often well-defined characteristic properties of a given material, the stress-free boundary conditions in the wake of the singular crack field are conceptually simple to both understand and implement. In contrast, frictional ruptures are considerably more complex entities. $\Gamma$ strongly depends on the details of how the system is loaded (e.g. $\Gamma$ couples to spatial variations of $\sigma_{y y}$ ) and any apparently minor contamination of the interface (e.g. a thin layer of lubricant) can increase its value by an order of magnitude or more. In this con- 
text, we presented a generic example of how $\Gamma$ may undergo extreme variations due to an effective layer of "interface contamination". In addition, whereas classic cracks, by definition, satisfy stress-free boundary conditions, it is not trivial that frictional cracks effectively can satisfy these conditions at all. Both the $\sigma_{x y}^{0}(x)$ and $\sigma_{x y}^{r e s}(x)$ are, in general, strongly varying in space while their difference, $\Delta \sigma_{x y}(x)$, which governs their dynamics, is generally an order of magnitude smaller than the mean values of both $\sigma_{x y}^{0}(x)$ and $\sigma_{x y}^{r e s}(x)$. In all of these examples, we have demonstrated that the fracture mechanics framework embodied in Eq. 4 indeed provides a surprisingly accurate quantitative description of the arrest of frictional ruptures. In particular, we have established a method to determine if, how and where a frictional rupture will arrest.

While fracture mechanics have been used previously in the context of frictional ruptures [Scholz, 2002; Rice, 1980; Ben-Zion, 2008; Ida, 1972; Dunham and Archuleta, 2004], we believe that we have convincingly demonstrated the validity of this approach in highly non-trivial experimental situations that have put these ideas to challenging (albeit successful) tests. Moreover, the case studies that we have presented demonstrate how the fracture mechanics framework can predict the behavior of a frictional system in many different practical configurations. Most of our results would be entirely counterintuitive were frictional motion to be described by simply the ratios of forces that are embodied within the classical notion of a macroscopic friction coefficient.

We would like to make a few comments regarding the validity of using a macroscopic static friction coefficient, defined as a ratio of applied forces, to describe rupture (or earthquake) propagation. Rupture nucleation is the key process responsible for the interface instability, i.e. the onset of motion. After nucleation, the rupture dynamics are dictated by the amount of excess elastic energy stored within the system prior to nucleation of an event [Svetlizky et al., 2017b]. As this quantity varies considerably (see e.g. Fig. 5a), so can the events that result. Indeed, experiments in simple systems such as described here can describe the entire broad spectrum of rupture events that are observed in natural faults (from slowly propagating ruptures to supershear events that approach the longitudinal wave speed of the material) [Ben-David et al., 2010; Kaproth and Marone, 2013; Svetlizky et al., 2017b; Xu et al., 2017]. What controls the formation of a nucleated "seed" crack along the frictional interface is still under debate [Uenishi and Rice, 2003; Lapusta and Rice, 2003; Rubin and Ampuero, 2005; Kaneko and Ampuero, 2011; Bar-Sinai et al., 2013; Latour et al., 2013; Viesca, 2016], however, rupture nucleation is experimentally observed to occur for a very wide range of applied stresses [Ben-David et al., 2010; Passelègue et al., 2013; Svetlizky et al., 2017b]. For this reason, a critical value of the loading, embodied in the macroscopic static friction coefficient, is an ill-defined quantity as it is neither characteristic of a material nor single valued for a given fault system. Although a well-defined value of the friction coefficient has been measured for different rocks [Byerlee, 1978], other experiments find a widespread of static friction coefficient values [Rabinowicz, 1992; Ben-David and Fineberg, 2011], that can be explained by the strong dependence of the rupture nucleation occurrence on the loading configuration. Note that, unlike a global critical force criterion, the "local" strength of the interface may well be a well-defined quantity. The peak interfacial strength is the peak value that stresses at the interface should reach in order for a rupture to propagate. In fact, various cohesive zone models have considered finite interfacial strengths to regularize the stress singularities associated with brittle fracture, as no stress divergence is expected to exist in nature ([Palmer and Rice, 1973] for instance).

We have seen, however, that the dynamic friction coefficient of a given system is, in these experiments, fairly constant and well defined - as is the closely related quantity $\sigma_{x y}^{r e s}(x)$. The many interesting recent experiments in which this quantity is measured [Tsutsumi and Shimamoto, 1997; Goldsby and Tullis, 2002; Di Toro et al., 2004, 2006; Reches and Lockner, 2010; Chang et al., 2012; Goldsby and Tullis, 2011] 
are therefore important in characterizing both the state of the interface (e.g. $\sigma_{x y}^{r e s}(x)$ in this formalism) as well as the physical mechanisms that serve to determine the explicit value of the fracture energy [Nielsen et al., 2016; Brantut and Viesca, 2017]. As we have shown, the residual stress is indeed a critical component in understanding how a rupture will propagate and/or arrest.

As the fracture mechanics based approach described above has now been validated, it can be utilized to determine the possible causes for a rupture arrest, and hence for an earthquake's size selection. The energy balance condition teaches us that, on one hand, arrest occurs because of either a lack of loading or a strong dissipation along a fault. Barriers of energy or stress can indeed suddenly arrest rupture propagation. On the other hand, rupture dynamics are controlled by the integrated drop of stress, e.g. through either loading or prior rupture histories. As a consequence, the stress profiles over an entire interface or fault should be considered in order to predict rupture arrest. Following this view, rupture arrest is deterministic when properly accounting for both loading and dissipation sources. These quantities, taken separately, cannot predict rupture dynamics.

We would like to make a brief comment about the role of either gouge or damage surrounding natural faults in earthquake rupture [Chang et al., 2012; Kaproth and Marone, 2013; Passelègue et al., 2013; Xu et al., 2017]. In the formalism described here, these quantities will have a direct influence on the value and possible spatial variation of the fracture energy, $\Gamma$. As long as the elastic energy within the system is stored at scales which are much larger than the scale of either the damage or gouge zones, fracture mechanics is blind to the structure of the dissipative zone (which is often called the "cohesive zone" or "process zone"). Only when such scale separation is lost will the detailed structure of these regions become apparent (for sub-Rayleigh ruptures). It is also for this reason that our relatively "simple" experimental system is entirely relevant for description of earthquake dynamics. In these experiments such a scale separation exists - as the elastic energy stored prior to rupture is contained in regions that are at least 4-5 orders of magnitude larger than the scale of cohesive zone (related to the surface roughness of a few $\mu \mathrm{m}$ 's). We have indeed shown that strain measurements located only a few mm's from the interface in our system are, essentially, entirely within the elastic (singular) region [Svetlizky and Fineberg, 2014].

This "blindness" of the fracture mechanics formalism to the structure of the dissipative zone can be used to advantage. If one is able to perform near-field measurements of the dynamic strains surrounding a sufficiently large propagating earthquake [Dunham and Archuleta, 2004], or equivalently the ground motion [Fukuyama and Suzuki, 2016; Kaneko et al., 2017], one can use the waveforms predicted by fracture mechanics (see e.g. Fig. 3) to quantitatively measure the value of the fracture energy of a fault directly - without the need to resort to estimations of the fracture energy and/or stress drops obtained from far-field seismic measurements. It would be, indeed, extremely interesting to directly compare such direct measurements of these important quantities to the values obtained by other, less direct, methods. This, of course, should be considered with some caution as the necessary conditions of the LEFM theory should be satisfied. In particular, a singular field with a K-dominance should exist at the rupture tip. The theory assumes measurements at an intermediate scale, larger than the cohesive zone size and short enough to ignore the contribution of far-field nonsingular terms. The energy balance condition should also be satisfied. If additional effects, such as thermal pressurization, plasticity of the damaged zone or propagation of additional ruptures, for example occur on fault boundaries, then the dissipation process due solely to the rupture propagation couldn't be isolated from other dissipative processes [Abercrombie and Rice, 2005] and could well be dependent on the state (e.g. instantaneous slip velocity) of the interface. In such cases, the fracture energy would not be independent of the rupture process. 
We believe that the use of the fracture paradigm for frictional behavior has opened a new window into understanding the fundamental behavior of the important processes that take place in both frictional sliding and earthquake dynamics. Potential applications abound, as frictional motion is of paramount importance in fields ranging from engineering and tribology to geodynamics and earthquake motion. The case studies presented here are just samples of a wide range of possible consequences of this description of friction that still wait to be explored.

\section{Acknowledgments}

This work was supported by the European Research Council (Grant No. 267256), the Israel Science Foundation (Grant No. 1523/15) and the US-Israel Binational Science Foundation (Grant No. 2016950) (all to I.S., E.B. and J.F.). E. B. acknowledges the support from the Lady Davis Trust. The analysis presented here is based on data available at this link: http://www.phys.huji.ac.il/ jay/JGRdatadirectory/Bayartdata.xlsx.

\section{References}

Abercrombie, R. E., and J. R. Rice (2005), Can observations of earthquake scaling constrain slip weakening?, Geophysical Journal International, 162(2), 406-424, doi: 10.1111/j.1365-246X.2005.02579.x.

Aldam, M., Y. Bar-Sinai, I. Svetlizky, E. A. Brener, J. Fineberg, and E. Bouchbinder (2016), Frictional sliding without geometrical reflection symmetry, Physical Review $X, 6(4)$.

Ampuero, J. P., J. Ripperger, and P. M. Mai (2006), Properties of dynamic earthquake ruptures with heterogeneous stress drop, American Geophysical Union.

Bar-Sinai, Y., R. Spatschek, E. A. Brener, and E. Bouchbinder (2013), Instabilities at frictional interfaces: Creep patches, nucleation, and rupture fronts, Physical Review E, 88(6), 060,403.

Bar-Sinai, Y., R. Spatschek, E. A. Brener, and E. Bouchbinder (2015), Velocitystrengthening friction significantly affects interfacial dynamics, strength and dissipation, Scientific Reports, 5, 7841.

Baumberger, T., C. Caroli, and O. Ronsin (2003), Self-healing slip pulses and the friction of gelatin gels, European Physical Journal E, 11(1), 85-93.

Bayart, E., I. Svetlizky, and J. Fineberg (2016a), Fracture mechanics determine the lengths of interface ruptures that mediate frictional motion, Nat Phys, 12, 166-170.

Bayart, E., I. Svetlizky, and J. Fineberg (2016b), Slippery but tough: The rapid fracture of lubricated frictional interfaces, Physical Review Letters, 116(19), 194,301, pRL.

Ben-David, O., and J. Fineberg (2011), Static friction coefficient is not a material constant, Physical Review Letters, 106 (25), 254,301.

Ben-David, O., G. Cohen, and J. Fineberg (2010), The dynamics of the onset of frictional slip, Science, 330(6001), 211-214.

Ben-Zion, Y. (2008), Collective behavior of earthquakes and faults: Continuumdiscrete transitions, progressive evolutionary changes, and different dynamic regimes, Reviews of Geophysics, 46(4), RG4006.

Beroza, G. C., and W. L. Ellsworth (1996), Properties of the seismic nucleation phase, Tectonophysics, 261, 209-227.

Bowden, F. P., and D. Tabor (2001), The Friction and Lubrication of Solids, 2 ed., Oxford Univ. Press, New York.

Brantut, N., and R. C. Viesca (2017), The fracture energy of ruptures driven by flash heating, Geophysical Research Letters, 44(13), 6718-6725, doi: 10.1002/2017GL074110, 2017GL074110. 
Braun, O. M., I. Barel, and M. Urbakh (2009), Dynamics of transition from static to kinetic friction, Physical Review Letters, 103(19), 194,301.

Brune, J. N. (1979), Implications of earthquake triggering and rupture propagation for earthquake prediction based on premonitory phenomena, Journal of Geophysical Research: Solid Earth, 84(B5), 2195-2198, doi:10.1029/JB084iB05p02195.

Byerlee, J. (1978), Friction of rocks, pure and applied geophysics, 116(4), 615-626, doi:10.1007/BF00876528.

Cappa, F., and J. Rutqvist (2011), Impact of co2 geological sequestration on the nucleation of earthquakes, Geophysical Research Letters, 38(17), doi: 10.1029/2011GL048487, 117313.

Chang, J. C., D. A. Lockner, and Z. Reches (2012), Rapid acceleration leads to rapid weakening in earthquake-like laboratory experiments, Science, 338(6103), 101-105, doi:10.1126/science.1221195.

Di Toro, G., T. Hirose, S. Nielsen, G. Pennacchioni, and T. Shimamoto (2006), Natural and experimental evidence of melt lubrication of faults during earthquakes, Science, 311 (5761), 647-649, doi:10.1126/science.1121012.

Di Toro, G., D. Goldsby, and T. Tullis (2004), Friction falls towards zero in quartz rock as slip velocity approaches seismic rates, NATURE, 427(6973), 436-439, doi: 10.1038 /nature02249.

Dieterich, J. H., and B. D. Kilgore (1996), Imaging surface contacts: Power law contact distributions and contact stresses in quartz, calcite, glass and acrylic plastic, Tectonophysics, 256(1-4), 219-239.

Dunham, E. M., and R. J. Archuleta (2004), Evidence for a supershear transient during the 2002 denali fault earthquake, Bulletin of the Seismological Society of America, 94(6), S256-S268.

Elliott, A. J., J. F. Dolan, and D. D. Oglesby (2009), Evidence from coseismic slip gradients for dynamic control on rupture propagation and arrest through stepovers, Journal of Geophysical Research: Solid Earth, 114(B2), doi: 10.1029/2008JB005969.

Ellsworth, W. L., and G. C. Beroza (1995), Seismic evidence for an earthquake nucleation phase, Science, 268(5212), 851-855.

Ellsworth, W. L., and G. C. Beroza (1998), Observation of the seismic nucleation phase in the ridgecrest, california, earthquake sequence, Geophysical Research Letters, 25(3), 401-404, doi:10.1029/97GL53700.

Freund, L., and A. Rosakis (1992), The structure of the near-tip field during transient elastodynamic crack growth, Journal of the Mechanics and Physics of Solids, 40(3), 699 - 719, doi:https://doi.org/10.1016/0022-5096(92)80010-N.

Freund, L. B. (1990), Dynamic Fracture Mechanics, Cambridge, New York.

Fukuyama, E., and W. Suzuki (2016), Near-fault deformation and dc during the 2016 mw7.1 kumamoto earthquake, Earth, Planets and Space, 68(1), 194, doi: 10.1186/s40623-016-0570-6.

Fukuyama, E., K. Tsuchida, H. Kawakata, F. Yamashita, K. Mizoguchi, and S. Xu (2017), Spatiotemporal complexity of 2-d rupture nucleation process observed by direct monitoring during large-scale biaxial rock friction experiments, Tectonophysics, doi:https://doi.org/10.1016/j.tecto.2017.12.023.

Galis, M., J. P. Ampuero, P. M. Mai, and F. Cappa (2017), Induced seismicity provides insight into why earthquake ruptures stop, Science Advances, 3(12), doi: 10.1126/sciadv.aap7528.

Goldsby, D. L., and T. E. Tullis (2002), Low frictional strength of quartz rocks at subseismic slip rates, Geophysical Research Letters, 29(17), 25-1-25-4, doi: 10.1029/2002GL015240.

Goldsby, D. L., and T. E. Tullis (2011), Flash heating leads to low frictional strength of crustal rocks at earthquake slip rates, Science, 334(6053), 216-218, doi:10.1126/science.1207902. 
Granick, S. (1991), Motions and relaxations of confined liquids, Science, 253(5026), 1374-1379.

Hamrock, B., S. Schmid, and B. Jacobson (2004), Fundamentals of fluid film lubrication, CRC Press.

Hirono, T., K. Tsuda, W. Tanikawa, J.-P. Ampuero, B. Shibazaki, M. Kinoshita, and J. J. Mori (2016), Near-trench slip potential of megaquakes evaluated from fault properties and conditions, Scientific Reports, 6, 28,184, doi:doi:10.1038/srep28184.

Ida, Y. (1972), Cohesive force across tip of a longitudinal-shear crack and griffiths specific surface energy, Journal of Geophysical Research, 77(20), 3796.

Kame, N., and T. Yamashita (1999), A new light on arresting mechanism of dynamic earthquake faulting, Geophysical Research Letters, 26(13), 1997-2000, doi: 10.1029/1999GL900410.

Kammer, D. S., M. Radiguet, J. P. Ampuero, and J. F. Molinari (2015), Linear elastic fracture mechanics predicts the propagation distance of frictional slip, Tribology letters, $57(3), 23$.

Kaneko, Y., and J.-P. Ampuero (2011), A mechanism for preseismic steady rupture fronts observed in laboratory experiments, Geophysical Research Letters, 38(21), L21,307, doi:10.1029/2011GL049953, 121307.

Kaneko, Y., E. Fukuyama, and I. J. Hamling (2017), Slip-weakening distance and energy budget inferred from near-fault ground deformation during the 2016 mw7.8 kaikōura earthquake, Geophysical Research Letters, 44(10), 4765-4773, doi: 10.1002/2017GL073681, 2017GL073681.

Kaproth, B. M., and C. Marone (2013), Slow earthquakes, preseismic velocity changes, and the origin of slow frictional stick-slip, Science (New York, N.Y.), 341(6151), $1229-32$.

Katano, Y., K. Nakano, M. Otsuki, and H. Matsukawa (2014), Novel friction law for the static friction force based on local precursor slipping, Scientific Reports, 4, 6324 .

Kilb, D., and J. Gomberg (1999), The initial subevent of the 1994 northridge, california, earthquake: Is earthquake size predictable?, Journal of Seismology, 3, 409-420.

Lapusta, N., and J. R. Rice (2003), Nucleation and early seismic propagation of small and large events in a crustal earthquake model, Journal of Geophysical ResearchSolid Earth, 108(B4), 2205.

Latour, S., A. Schubnel, S. Nielsen, R. Madariaga, and S. Vinciguerra (2013), Characterization of nucleation during laboratory earthquakes, Geophysical Research Letters, 40(19), 5064-5069.

Lozos, J. C. (2016), A case for historic joint rupture of the san andreas and san jacinto faults, Science Advances, 2(3), doi:10.1126/sciadv.1500621.

Maegawa, S., A. Suzuki, and K. Nakano (2010), Precursors of global slip in a longitudinal line contact under non-uniform normal loading, Tribology letters, 38(3), $313-323$

McLaskey, G. C., B. D. Kilgore, and N. M. Beeler (2015), Slip-pulse rupture behavior on a $2 \mathrm{~m}$ granite fault, Geophysical Research Letters, 42(17), 7039-7045, doi:10.1002/2015GL065207, 2015GL065207.

Nielsen, S., E. Spagnuolo, S. A. F. Smith, M. Violay, G. Di Toro, and A. Bistacchi (2016), Scaling in natural and laboratory earthquakes, Geophysical Research Letters, 43(4), 1504-1510, doi:10.1002/2015GL067490, 2015GL067490.

Ohnaka, M. (2000), A physical scaling relation between the size of an earthquake and its nucleation zone size, Pure and Applied Geophysics, 157(11), 2259-2282.

Ohnaka, M. (2013), The Physics of Rock Failure and Earthquakes, 279 pp., Cambridge University Press.

Ohnaka, M., and Y. Kuwahara (1990), Characteristic features of local breakdown near a crack-tip in the transition zone from nucleation to unstable rupture during stick-slip shear failure, Tectonophysics, 175(1), 197 - 220, doi: https://doi.org/10.1016/0040-1951(90)90138-X, earthquake Source Processes. 
Okubo, P. G., and J. H. Dieterich (1981), Fracture energy of stick-slip events in a large scale biaxial experiment, Geophysical Research Letters, 8(8), 887-890, doi: 10.1029/GL008i008p00887.

Olson, E. L., and R. M. Allen (2005), The deterministic nature of earthquake rupture, Nature, 438(7065), 212-215.

Palmer, C., A., and R. Rice, J. (1973), The growth of slip surfaces in the progressive failure of over-consolidated clay, Proc. R. Soc. Lond. A, 332, 527-548.

Passelègue, F. X., A. Schubnel, S. Nielsen, H. S. Bhat, and R. Madariaga (2013), From sub-rayleigh to supershear ruptures during stick-slip experiments on crustal rocks, Science, 340(6137), 1208-1211.

Peng, Z., and J. Gomberg (2010), An integrated perspective of the continuum between earthquakes and slow-slip phenomena, Nature Geosci, 3(9), 599-607, $10.1038 /$ ngeo940.

Rabinowicz, E. (1992), Friction coefficients of noble metals over a range of loads, Wear, 159 (1), 89 - 94, doi:https://doi.org/10.1016/0043-1648(92)90289-K.

Radiguet, M., D. S. Kammer, P. Gillet, and J. F. Molinari (2013), Survival of heterogeneous stress distributions created by precursory slip at frictional interfaces, Physical Review Letters, $111(16), 164,302$.

Read, B. E., and J. C. Duncan (1981), Measurement of dynamic properties of polymeric glasses for different modes of deformation, Polymer Testing, 2(2), 135-150.

Reches, Z., and D. A. Lockner (2010), Fault weakening and earthquake instability by powder lubrication, NATURE, 467(7314), doi:10.1038/nature09348.

Rice, J. R. (1980), The mechanics of earthquake rupture, in Physics of the Earth's Interior, edited by A. M. Dziewonski and E. Boschi, pp. 555-649, Italian Physical Society and North-Holland Publ. Co.

Rubin, A. M., and J.-P. Ampuero (2005), Earthquake nucleation on (aging) rate and state faults, J. Geophys. Res., 110(B11312).

Rubino, V., A. J. Rosakis, and N. Lapusta (2017), Understanding dynamic friction through spontaneously evolving laboratory earthquakes, Nature Communications, 8, 15,991, doi:10.1038/ncomms15991.

Rubinstein, S., G. Cohen, and J. Fineberg (2006), Contact area measurements reveal loading-history dependence of static friction, Physical Review Letters, 96(25), 256,103 .

Rubinstein, S. M., G. Cohen, and J. Fineberg (2004), Detachment fronts and the onset of dynamic friction, Nature, 430(7003), 1005-1009, nATURE.

Rubinstein, S. M., G. Cohen, and J. Fineberg (2007), Dynamics of precursors to frictional sliding, Physical Review Letters, 98(22), 226,103.

Rubinstein, S. M., G. Cohen, and J. Fineberg (2009), Visualizing stick-slip: experimental observations of processes governing the nucleation of frictional sliding, Journal of Physics D: Applied Physics, 42.

Scheibert, J., and D. K. Dysthe (2010), Role of friction-induced torque in stick-slip motion, Epl, 92(5), 54,001.

Scholz, C. (2002), The mechanics of earthquakes and faulting, 2nd ed., Cambridge University Press, Cambridge.

Steacy, S. J., and J. McCloskey (1998), What controls an earthquakeś size? results from a heterogeneous cellular automaton, Geophysical Journal International, 133, F11-F14.

Svetlizky, I., and J. Fineberg (2014), Classical shear cracks drive the onset of dry frictional motion, Nature, 509(7499), 205-208.

Svetlizky, I., D. P. Munoz, M. Radiguet, D. S. Kammer, J.-F. Molinari, and J. Fineberg (2016), Properties of the shear stress peak radiated ahead of rapidly accelerating rupture fronts that mediate frictional slip, Proceedings of the National Academy of Sciences of the United States of America, 113(3), 542-547. 
Svetlizky, I., E. Bayart, G. Cohen, and J. Fineberg (2017a), Frictional resistance within the wake of frictional rupture fronts, Physical Review Letters, 118(23).

Svetlizky, I., D. S. Kammer, E. Bayart, G. Cohen, and J. Fineberg (2017b), Brittle fracture theory predicts the equation of motion of frictional rupture fronts, Physical Review Letters, 118(12).

Tada, H., P. C. Paris, and G. R. Irwin (2000), The stress analysis of cracks handbook, American Society of mechanical engineers, [S.l.].

Taloni, A., A. Benassi, S. Sandfeld, and S. Zapperi (2015), Scalar model for frictional precursors dynamics, Scientific Reports, 5, 8086.

Tromborg, J., J. Scheibert, D. S. Amundsen, K. Thogersen, and A. Malthe-Sorenssen (2011), Transition from static to kinetic friction: Insights from a 2d model, Physical Review Letters, 107(7), 074,301.

Tsutsumi, A., and T. Shimamoto (1997), High-velocity frictional properties of gabbro, Geophysical Research Letters, 24(6), 699-702, doi:10.1029/97GL00503.

Uenishi, K., and J. R. Rice (2003), Universal nucleation length for slip-weakening rupture instability under nonuniform fault loading, Journal of Geophysical Research: Solid Earth, 108(B1), 2042, doi:10.1029/2001JB001681.

Viesca, R. C. (2016), Stable and unstable development of an interfacial sliding instability, Phys. Rev. E, 93, 060,202, doi:10.1103/PhysRevE.93.060202.

Villey, R., E. Martinot, C. Cottin-Bizonne, M. Phaner-Goutorbe, L. Leger, F. Restagno, and E. Charlaix (2013), Effect of surface elasticity on the rheology of nanometric liquids, Phys. Rev. Lett., $111(21)$, 215,701.

Wesnousky, S. (2006), Predicting the endpoints of earthquake ruptures, Nature, $444(358)$.

Xia, K., A. J. Rosakis, and H. Kanamori (2004), Laboratory earthquakes: The subraleigh-to-supershear rupture transition, Science, 303(19), 1859-1861.

$\mathrm{Xu}$, S., and Y. Ben-Zion (2017), Theoretical constraints on dynamic pulverization of fault zone rocks, Geophysical Journal International, 209(1), 282-296.

Xu, S., E. Fukuyama, H. Yue, and J.-P. Ampuero (2016), Simple crack models explain deformation induced by subduction zone megathrust earthquakes, Bulletin of the Seismological Society of America, 106(5), 2275-2289.

$\mathrm{Xu}$, S., E. Fukuyama, F. Yamashita, K. Mizoguchi, S. Takizawa, and H. Kawakata (2017), Strain rate effect on fault slip and rupture evolution: Insight from meter-scale rock friction experiments, Tectonophysics, doi: https://doi.org/10.1016/j.tecto.2017.11.039. 